\title{
Mucosal Associated Invariant T (MAIT) Cell Responses Differ by Sex in COVID-19
}

3 Chen $\mathrm{Yu}^{1, \dagger}$, Sejiro Littleton ${ }^{1,2, \dagger}$, Nicholas Giroux ${ }^{3}$, Rose Mathew ${ }^{1}$, Shengli Ding ${ }^{3}$, Joan Kalnitsky ${ }^{1}$,

4 Elizabeth W. Petzold ${ }^{5}$, Hong Chung ${ }^{3}$, Grecia Rivera Palomino ${ }^{3}$, Tomer Rotstein ${ }^{3}, \mathrm{Rui} \mathrm{Xi}^{3}$, Emily R.

$5 \mathrm{Ko}^{5,6}$, Ephraim L. Tsalik ${ }^{5,7,8}$, Gregory D. Sempowski ${ }^{9}$, Thomas N. Denny ${ }^{9}$, Thomas W. Burke ${ }^{5}$, Micah T.

6 McClain ${ }^{5,7-8}$, Christopher W. Woods ${ }^{5,7-9}$, Xiling Shen ${ }^{3}$, Daniel R. Saban ${ }^{1,2, *}$

\section{Affiliations:}

$8 \quad{ }^{1}$ Department of Ophthalmology, Duke University School of Medicine, Durham, NC, USA.

92 Department of Immunology, Duke University School of Medicine, Durham, NC, USA.

$10{ }^{3}$ Department of Biomedical Engineering, Pratt School of Engineering, Duke University, Durham, NC,

11 USA.

$12{ }^{4}$ Department of Cell Biology, Duke University Medical School, Durham, United States.

$13{ }^{5}$ Center for Applied Genomics and Precision Medicine, Duke University, Durham, NC, USA

$14{ }^{6}$ Duke Department of Medicine, Duke University School of Medicine, Durham, NC, USA

$15{ }^{7}$ Durham Veterans Affairs Health Care System, Durham, NC, USA

$16{ }^{8}$ Division of Infectious Diseases, Duke University Medical Center, Durham, NC, USA

$17{ }^{9}$ Duke Human Vaccine Institute, Duke University Medical Center, Durham, NC, USA

18 TThese authors contributed equally to this work.

19 "Corresponding author. E-mail: daniel.saban@duke.edu 


\section{ABSTRACT}

21 Sexual dimorphisms in immune responses contribute to coronavirus disease 2019 (COVID-19)

22 outcomes, yet the mechanisms governing this disparity remain incompletely understood. We carried out

23 sex-balanced sampling of peripheral blood mononuclear cells from confirmed COVID-19 inpatients and

24 outpatients, uninfected close contacts, and healthy controls for 36-color flow cytometry and single cell

25 RNA-sequencing. Our results revealed a pronounced reduction of circulating mucosal associated

26 invariant T (MAIT) cells in infected females. Integration of published COVID-19 airway tissue datasets

27 implicate that this reduction represented a major wave of MAIT cell extravasation during early infection

28 in females. Moreover, female MAIT cells possessed an immunologically active gene signature, whereas

29 male counterparts were pro-apoptotic. Collectively, our findings uncover a female-specific protective

30 MAIT profile, potentially shedding light on reduced COVID-19 susceptibility in females. 


\section{MAIN TEXT}

Severe Acute Respiratory Syndrome Coronavirus 2 (SARS-CoV-2) has led to a global pandemic of Coronavirus Disease 2019 (COVID-19) and a death toll of more than over 1.4 million people and rising (1). Among reported sex disaggregated data, males are disproportionately affected by SARS-CoV-2, with a higher incidence of cases, mortality, and morbidity (2). This follows a similar trend toward higher case fatality rates for males in Severe Acute Respiratory Syndrome Coronavirus (SARS-CoV) and Middle East Respiratory Syndrome Coronavirus (MERS-CoV), as well as with experiments using SARS-CoV mouse models (2-6). Sex differences in the immune response are thought to be a key contributing factor to these coronavirus disease outcomes, agreeing with the current body of knowledge that innate and adaptive immune responses are substantially altered across sex (7-10). Specific to SARS-CoV-2 infection, responses of both lymphocytes and myeloid cells were shown to be associated with COVID-19 outcomes (11-19). Correspondingly, a recent study on sex differences in COVID-19 immune responses uncovered an association between poor disease outcomes in males and weak T cell responses in both $\mathrm{CD}^{+}$and $\mathrm{CD}^{+}$compartments, whereas poor outcomes in females were associated with high innate immune cytokines, tumor necrosis factor superfamily (TNFSF)-10 and interleukin (IL)-15 (20). The sex differences elucidated in this seminal study further cement the need to better understand the mechanisms governing sex-specific susceptibility to SARS-CoV-2.

In the current study, we carried out sex-balanced sampling of peripheral blood mononuclear cells (PBMCs) from COVID-19 patients and control subjects for 36-color flow cytometry and single cell RNAsequencing (scRNA-seq) analyses. A total of 88 samples were analyzed from 45 individuals. Details on subject demographics and sample information are summarized in (Fig. 1A, table 1 and S1). Briefly, we analyzed samples from 28 patients with COVID-19 as confirmed by a positive SARS-CoV-2 PCR and/or IgG seroconversion. These included 9 inpatient subjects (20\%), 7 requiring intensive care, henceforth referred to as "hospitalized." An additional 19 subjects were identified in outpatient settings 
longitudinally (a range 1-28 days) including pre- and post- anti-SARS-CoV-2 immunoglobin (IgG) seroconversion. The dates of symptom onset for all confirmed COVID-19 subjects were recorded at enrollment, providing an illness range of 1-40 days. We also recorded symptom severity, obtained via investigator survey on 39 symptoms related to COVID-19 (see Methods). Additionally, we included 7 subjects $(15.6 \%)$ henceforth referred to as "exposed," who were also sampled at multiple timepoints.

These subjects, despite being close contacts of infected individuals, remained with negligible symptom scores, were negative for SARS-CoV-2 by PCR, and did not demonstrate detectable anti-SARS-CoV-2 IgG for at least 2 months after enrollment. Lastly, we included a group of 10 "healthy" subjects (22.2\%) who were enrolled prior to the pandemic in 2019 and did not show any symptoms associated with COVID-19 or other respiratory illness (21).

\section{Immune Profiling of COVID-19 Patient PBMCs Reveals Sex Differences in CD8+ Lymphocytes}

With these flow cytometry data (Table S2), we generated a map of immune cell populations and their subsets by down-sampling to 3,000 viable CD45+ singlets per sample and concatenated all data for Uniform Manifold Approximation and Projection (UMAP) (22) and unbiased clustering via Flow Selfannotation of major PBMC populations, including $\mathrm{CD}^{+}$and $\mathrm{CD}^{+}(\alpha \beta) \mathrm{T}$ cells, $\gamma \delta \mathrm{T}$ cells, B cells, plasmablasts, natural killer (NK) cells, monocytes (MO), and dendritic cells (DC), and confirmed by manual analysis (Fig. 1B, and fig. S1, A to E). Sub-populations were also annotated in this manner, such as $\mathrm{CD}_{45 \mathrm{RA}}{ }^{+} \mathrm{CD} 27^{+} \mathrm{CCR} 7^{+}$naive, $\mathrm{CD} 45 \mathrm{RA}^{-} \mathrm{CCR} 7^{+}$central memory (CM), CD45RA- CCR7effector memory (EM) and CD45RA ${ }^{+}$CD27 ${ }^{-}$CCR7- terminally differentiated effector memory (EMRA) $\mathrm{CD}^{+} \mathrm{T}$ cells, as well as CD8 ${ }^{+} \mathrm{CD} 161^{\mathrm{hi}} \mathrm{T}$ cells and other indicated sub-populations (Fig. 1B, and fig. hospitalized patients were detected (Fig. 1B), despite using a PBMC isolation protocol. 
We next set out to examine our flow cytometry dataset for immune populations that exhibited major quantitative changes in COVID-19. Our first strategy was to stratify the data by disease severity (i.e.,

81 healthy, exposed, infected, and hospitalized). We noted that samples from hospitalized patients had

82 substantially fewer PBMCs suggestive of lymphopenia (24). Manual gating of all flow cytometry events

83 was performed for this analysis. Our results showed differences in B cells (naïve, IgD+ non-class

84 switch, and plasmablasts); natural killer (NK) cells (CD56 ${ }^{\text {lo }}$ populations); DCs (CD141 ${ }^{+}, \mathrm{CD}^{+} \mathrm{C}^{+}$, and

85 pDCs); monocytes (classical, intermediate and nonclassical); $\mathrm{CD}^{+}(\mathrm{EM})$ and $\mathrm{CD}^{+}(\mathrm{EM}) \alpha \beta \mathrm{T}$ cells

86 (Fig. S2). Interestingly, our data also revealed a high statistical significance $(p=0.0006)$ in CD8 ${ }^{+}$

87 CD161 ${ }^{\text {hi }}$ T cells (Fig. S2) prompting us to look closer at these cells. Regarding annotation of this

CD161 ${ }^{\text {hi }}$ cluster, because the overwhelming majority of events are low to negative for CD56 and for T-

cell receptor (TCR)-y $\delta$ (Fig. 1C), the phenotype is largely consistent with mucosal associated invariant

$\mathrm{T}$ (MAIT) cells, but not NKT or $\gamma \delta$ T cells. This designation is congruent with recent work in COVID-19

PBMCs (25-27). We therefore conclude that the frequencies of certain myeloid and lymphocyte populations are affected in COVID-19, including a major effect on CD8 ${ }^{+}$CD161 ${ }^{\text {hi }} \mathrm{T}$ cells.

Knowing that the overwhelming majority of the $C D 8^{+} C D 161^{\text {hi }}$ population (henceforth referred to as CD161 ${ }^{\text {hi }}$ ) in our and others' datasets (25-27) is likely comprised of MAIT cells, we performed a more focused analysis of this cluster in COVID-19 (Fig. S3A). First, we analyzed their frequencies by disease severity using the samples taken within 3 days of enrollment, the timepoint most proximal to the initial symptom score recordings. Results were displayed via UMAP contour plots, revealing a reduction in these cells in the SARS-CoV-2 settings (Fig. 1D). Manual gating from all flow cytometry events revealed a significant reduction when comparing healthy $(p=0.0036)$ or exposed $(p=0.0488)$ subjects versus hospitalized subjects, as well as a negative correlation $(p=0.0002)$ with disease severity (Fig. 1E and Fig. S3B). We also characterized the frequencies of CD161 $1{ }^{\text {hi }}$ cells stratified by time post symptom 102 onset, including early ( $\leq 14$ days), middle (15 to 21 days), and late ( $>21$ days) timepoints. In addition, 103 we separated the data by sex given the known sex differences in immune responses in COVID-19 (2, 
104 20). Results showed that within the $\mathrm{CD}^{+}$compartment of healthy subjects, females had greater

105 frequencies of CD161 ${ }^{\text {hi }}$ cells relative to males, whereas males had greater frequencies of $C D 8^{+}$memory 106 T cells (combined EMRA, EM and CM) (Fig. 1F, and G). No obvious changes of naïve CD8 ${ }^{+}$T were 107 found (Fig. S3C). While the memory cell predominance in males was preserved at all timepoints, the 108 greater abundance of CD161 ${ }^{\text {hi }}$ cells in females was lost at early and middle timepoints (albeit not in late 109 disease). The loss of this difference was due to a precipitous drop of CD161 ${ }^{\text {hi }}$ cells in females at early

110 and middle timepoints (Fig. 1H). Lastly, we stratified data from confirmed COVID-19 patients by

111 seroconversion status. This showed CD16 $1^{\text {hi }}$ cells were higher in females relative to males prior to

112 seroconversion, whereas $\mathrm{CD}^{+}$memory cells were higher in males in seroconverted subjects (Fig. 1I).

113 Taken together, we identified a female-specific decline in circulating CD161 ${ }^{\text {hi }}$ cell frequencies upon exposure/infection of SARS-CoV-2. This sex-specific reduction may be due to extravasation into airway 115 tissues, thereby suggesting a key sex-specific role for these CD161 ${ }^{\text {hi }}$ cells in COVID-19.

scRNA-seq of PBMCs Implicates Involvement of CD161 ${ }^{\text {hi }}$ Lymphocyte Responses in COVID-19

117 Given the potentially important role for CD161 ${ }^{\text {hi }}$ cells in COVID-19, we sought to further characterize 118 this population by scRNA-seq (10x Genomics). We analyzed 48 different PBMC samples from 24 119 subjects across all groups (Table 1 and table S1). Data were processed using Seurat 3 package (28) 120 and subsequent transcript-based annotation was carried out (Fig. 2A, fig. S4, A and B). Focusing on 121 the T cells in the data (Fig. 2B and table S3), we were able to identify CD161 ${ }^{\text {hi }}$ cells in a single cluster 122 containing high $K L R B 1$ (i.e., CD161) expression, and co-expression of $C D 3 D$ and $C D 8 A$, as well as 123 TRAV1-2 (Fig. 2C and fig. S4B), which encodes the Va7.2 invariant TCR alpha chain on MAIT cells. 124 Grouping these data by disease severity showed that hospitalized patients had lower frequencies of $\mathrm{T}$ 125 cells, including CD16 $1^{\text {hi }}$ cells (Fig. 2D), agreeing with our flow cytometry findings and consistant with 126 reported lymphopenia in severe COVID-19 patients (11, 20, 29-32). Also showing the same trend as 127 our flow cytometry data was the high frequency of CD161 ${ }^{\text {hi }}$ cells in healthy females (Fig. 2E), although 128 it did not reach statistical significance due to the variations between healthy females and males. Next, 
129 to address the functional role of this CD161 ${ }^{\text {hi }}$ cluster in COVID-19, we performed gene enrichment 130 analysis using differentially expressed genes (DEGs). With several top ranked hits consisting of

131 immune pathways and an estrogen-dependent pathway (Fig. 2F), our results inferred a sex-specific

132 immune response of these CD161 ${ }^{\text {hi }}$ cells in COVID-19. To further characterize functional inferences, we 133 applied the CellphoneDB package (33) and analyzed ligand-receptor interactions with monocyte

134 clusters within our data (Fig. S5, A and B), given the critical link that was previously published between 135 monocyte activation in COVID-19 outcomes (12, 13, 20, 34). Our results inferred unique interactions 136 between $C D 161^{\text {hi }}$ cells and monocytes with the following gene-pairs: KLRB1_CLEC2D, CCL5_CCR1, 137 CXCR6_CXCL16, and IL18_IL-18R (Fig. S5, C and D). Moreover, the number of interaction-counts of 138 monocytes was the most abundant with the CD161 $1^{\text {hi }}$ cluster relative to all major T cell populations (Fig. 139 S5E). Taken together, these transcriptome findings further support a significant role for circulating 140 CD161 ${ }^{\text {hi }}$ cells in the SARS-CoV-2 immune response.

\section{Sex-Specific Differences of Circulating MAIT cells in COVID-19}

142 To analyze our scRNA-seq dataset for potential sex differences in circulating CD161 ${ }^{\text {hi }}$ cells, we first 143 sought to examine for phenotypic heterogeneity within this population. To do this, we performed a 144 focused sub-cluster analysis, which generated 3 distinct clusters (Fig. 3A). However, the added 145 resolution revealed a cluster that expressed $T R D C$, encoding the constant region of the $\delta$ chain 146 expressed by $\gamma \delta$ T cells (Fig. 3B) and thereby excluded from subsequent analyses. By contrast, the 147 other two clusters had higher KLRB1 expression, as well as TRAV1- 2 (Fig. 3B), therefore referred to 148 here as MAIT $\alpha$ and MAIT $\beta$ clusters. Of note, these 2 clusters make up approximately $80 \%$ of CD161 hi 149 PBMCs, which is consistent with the previous report of circulating MAIT cell frequencies (25). Our 150 results showed that the MAIT $\alpha$ cluster possessed upregulated genes associated with cytotoxic T cells 151 (GNLY,CD8A, CD8B), migration/adhesion (CXCR4, ITGB2), and cytokine signaling (IRF1, B2M, $152 N F K B I A, J U N B, F O S)$ (Fig. 3C and table S4). The MAIT $\beta$ cluster was enriched for genes of ribosomal 153 proteins, apoptosis (BAX, STUB1) and the linker histone $\mathrm{H} 1$ associated with apoptosis $(H I S T 1 H 1 C$, 
HIST1H1D, HIST1H1E) (Fig. 3C and table S4). Gene enrichment analysis further supported a

155 functional dichotomy for $\alpha$ and $\beta$ clusters. Whereas MAIT $\alpha$ was enriched with several immune process 156 pathways (e.g., IFN-y, and IL-4 and IL-13 signaling, as well as antigen processing and presenting), 157 MAIT $\beta$ was enriched in cellular responses to external stimuli, metabolism of RNA, viral infection, and 158 programmed cell death, but not immune processes (Fig. 3, D and E, and table S5). Hence our results 159 suggest MAIT cell heterogeneity, with the MAIT $\alpha$ signature representing an immunologically 160 poised/active phenotype, while the MAIT $\beta$ signature represents a stressed/apoptotic phenotype.

161 Last for this series of experiments, we sought to determine the dynamics of the two phenotypically 162 distinct clusters by sex over the COVID-19 disease course. By first grouping our data by severity, we 163 found that MAIT $\alpha$ was the major phenotype in healthy individuals, while MAIT $\beta$ predominated in 164 exposed and infected groups (Fig. 3, F and G). There was a noted exception for hospitalized patients 165 (Fig. 3F), bearing very few cells as seen in our flow cytometry data, consistent with lymphopenia that 166 occurs in severe COVID-19 (24). We then grouped our data by time post symptom onset, as we 167 detailed earlier with our flow cytometry data. Results showed that relative to healthy subjects, MAIT $\alpha$ 168 percentages were lower in early, middle, and late timepoints, whereas MAIT $\beta$ demonstrated the converse (Fig. 3H). When stratified by sex, we found that MAIT cell frequencies were higher in healthy females (Fig. 3, I and J), corroborating our flow cytometry results. These cells in healthy females were

171 skewed toward the MAIT $\alpha$ cluster, whereas the few cells present in healthy males consisted mostly of 172 MAIT $\beta$ (Fig. 3I). However, this difference was lost in exposed/infected setting, where both sexes were 173 comprised mostly of MAIT $\beta$ (Fig. 3J). Nonetheless, MAIT $\beta$ percentages were statistically greater in 174 females in late disease (Fig. 3J), which reflects the increased MAIT cells during late infection in 175 females as shown in our flow cytometry findings. Regarding expression of CD69, a T cell activation 176 marker, we did not observe major differences across cluster or sex, but did observe elevated 177 expression in the hospitalized group (Fig. S6, A to D). This possibly suggests an altered MAIT cell 
178 response in hospitalized patients $(25-27,35)$. In short, these results reveal sex specific MAIT cell

179 differences at the quantitative and phenotypic levels in health and COVID-19.

\section{Respiratory Tract MAIT Cell Responses Differ by Sex in COVID-19}

181 To assess potential sex-specific differences in MAIT cells at the tissue level in COVID-19 patient

182 airways, we utilized published scRNAseq datasets of bronchoalveolar lavage fluid (BALF) (36) and of 183 nasopharyngeal swab (NPS) (37). Beginning our analysis with the BALF dataset, we identified the 184 MAIT cell cluster by expression of TRAV1-2, CD3D, KLRB1 and SLC4A10 (Fig. 4, A to C). With this 185 annotation, we found a significant increase $(\mathrm{p}=0.0188)$ of MAIT cells in COVID-19 patients relative to 186 normal controls and a higher MAIT cell frequency $(p=0.0332)$ in females relative to males among 187 COVID-19 subjects (Fig. 4D). This detection of increased MAIT cells in female BALF, along with the 188 drop in these cells we observed in female peripheral blood, suggest a female dominant extravasation of MAITs in COVID-19.

190 To perform the same analysis with NPS samples, we integrated the T cell data from NPS with those

191 from BALF to identify MAIT cells in NPS (Fig. 4, E and F), since TCR genes were not aligned in the 192 NPS dataset (37). With this annotation, we quantified MAIT cell frequencies in the NPS dataset, again 193 observing a significant increase ( $p=0.0139)$ in COVID-19 patients (Fig. 4G). We also analyzed the data 194 across severity, observing a significant increase $(p=0.0038)$ in moderate subjects relative to the normal 195 and a decrease $(p=0.0366)$ relative to critical COVID-19 subjects (Fig. $4 \mathbf{H}, \mathbf{I})$. We could not perform the 196 same analysis by sex due to insufficient number of female samples (Fig. 4 I). Nonetheless, these 197 results match the reduced circulating MAIT cell frequencies seen in our hospitalized subjects, together 198 suggesting that a lymphopenic state which occurs in severe COVID-19 impacts MAIT cells, consistent 199 with other reports $(25-27,38)$.

200 In a final experiment, we sought to characterize MAIT cell transcriptomes by sex in the BALF and NPS 201 datasets and determine whether these cells resembled $\alpha$ and $\beta$ phenotypes we identified in circulating 
MAIT cells. Cluster analysis was not warranted here given low cell numbers in these datasets. Instead, we leveraged gene modules derived from our respective $\alpha$ and $\beta$ clusters of circulating MAIT cells.

We found that MAIT cells in BALF and NPS data were largely skewed toward the $\beta$ module, with

minimal sex differences (Fig. S7A). However, when we directly examined differentially expressed genes (the DEGs between sex, we were able to detect sex differences associated with $\alpha$ and $\beta$ phenotypes. Specifically, in BALF, we found increased IL7R expression in females (Fig. 4J) and other

IL-7 signaling associated genes (CISH and SOCS1) (Fig. 4K). Given the critical role of this signaling in

T cell survival, we explored additional pathway genes, finding that female MAIT cells had upregulated anti-apoptotic genes (BCL2 and FOXP1) and downregulated pro-apoptotic genes (BAX and CASP3)

(Fig. S7, B and C). Also observed in female cells was upregulated anti-proliferative genes (CDKN1B

213 able to find other sex differences, including increased expression of several transcription factors (KLF2,

214 MYC, and CEBPD) (Fig. 4L). Conversely, male cells had higher expression of CCL2 (Fig. 4 M), which 215 has been linked to COVID-19 immunopathology (39). In short, our results infer sex differences at the 216 qualitative level in COVID-19, with female MAIT cells possessing a pro-survival and immunologically 217 active phenotype.

\section{DISCUSSION}

219 Despite the knowledge of sex differences in the immune response as an underlying factor in COVID-19 220 disease outcomes, the sexual dimorphic responses of MAIT cells, an unconventional T cell population 221 deemed important in this disease, remained unknown. We now demonstrate that MAIT cells in females 222 are quantitatively and qualitatively more robust in the SARS-CoV-2 setting, potentially helping 223 understand the immunological reasons for reduced COVID-19 susceptibility in females.

224 Our finding that MAIT recruitment to airway tissues may be more robust in COVID-19 females was 225 aided first by our observation of higher circulating MAIT cell frequencies in females in the healthy 
setting. This difference can be explained by the rate of physiological aging-related attrition of MAIT cells that is substantially less pronounced in female blood (40-42). The resultant higher frequencies in circulation enabled us to readily uncover the precipitous percentage drop we saw with MAIT cells relative to exposed/infected females. In trying to elucidate the potential cause of this drop, we

230 considered two possible scenarios: 1) lymphopenia and 2) extravasation, which are not necessarily

231 mutually exclusive. For the former, it is accepted that lymphopenia is associated with severe COVID-19

232 infections $(24,32,43,44)$, which which agrees with our observations in our hospitalized group

233 (comprised of $77.8 \%$ intensive care patients). Similarly, lymphopenia could partially explain the

234 reduction in MAIT cells described by Jouan et al in a study of male-dominated samples from critically ill

235 COVID-19 patients (27), though extravasation also likely occurred. In our study, however, we

236 demonstrated that circulating MAIT frequencies drop in our infected outpatient group. As these subjects

237 were not critically ill, our findings point to extravasation as a major reason for the sex-specific drop in

238 circulating MAIT frequencies. The same pattern may also exist in several other studies $(25,26,35)$. For

239 example, while Parrot et al (25) also demonstrated that circulating MAIT cells are reduced in moderate

240 COVID-19 patients relative to healthy subjects in aggregated data, it is possible that the healthy female

241 frequencies contributed to reaching the statistical difference. Further supporting our conclusion, we

242 were able to show with publicly available scRNA-seq data from COVID-19 BALF samples (36) that

243 females in that study had an increased MAIT cell percentage relative to males, allowing us to conclude

244 that MAIT cell extravasation during COVID-19 may be quantitatively more robust in females.

245 Our results also suggest that MAIT cells may be qualitatively superior in females, with respect to anti-

246 viral immune activity in COVID-19. Leading us to this conclusion, our scRNA-seq analysis of patient

247 PBMCs revealed two distinct clusters of MAIT cells, referred to here as MAIT $\alpha$ and MAIT $\beta$. The $\alpha$

248 cluster was enriched for various immune pathways, such as IFN- $\gamma$ signaling, inferring a capacity for

249 anti-viral immune function. In contrast, the $\beta$ cluster was enriched for cell stress and apoptosis

250 pathways, inferring a frail phenotype roughly similar to a previously described population of double 
negative MAIT cells $(45,46)$. We showed in the healthy setting that MAIT cells in females were skewed

252 toward the $\alpha$ cluster, whereas males comprised the $\beta$ cluster. Though from these results it could be 253 presumed that the $\alpha$ cluster should be overrepresented in COVID-19 airways of females, this was not

254 the case in the BALF. However, we reasoned that such a finding would be very difficult to make for two main reasons. First, extravasated MAIT cells with an $\alpha$-phenotype would be restricted to the early wave of recruitment, since circulating cells are almost completely skewed to the $\beta$ module in exposed/infected 257 individuals. Second, a certain level of transcriptional reprogramming would occur upon immune cell extravasation into the tissue and potentially again upon accessing the alveolar space. Still, we were able to show in BALF that certain gene patterns remained consistent with the $\alpha$ signature in females versus males. In addition, our finding that female BALF samples had quantitatively more MAIT cells gives further credence that differences revealed in blood would likewise extend to the tissue.

In summary, we conclude that MAIT cells in females are quantitatively and qualitatively distinct from males and we surmise that this distinction provides a protective advantage in the SARS-CoV-2 setting. Indeed, females in general tend to have elevated frequencies of circulating MAIT cells, also gleaned by 265 large independent studies with European (40), South Korean (41) and Chinese populations (42).

266 Further supporting this argument, it has now been recorded that adult COVID-19 fatality rates trend 267 less in females at all ages across 39 different countries, including in North America, Europe, and Asia 268 where MAIT cell frequencies trend higher in females (2). These points also argue against the possibility 269 that an immunologically more robust MAIT cell response has a net negative effect, for example, by 270 immunological misfiring (20) or cytokine storm related immunopathology (39). However, one open 271 question that our findings now raise is whether males in our study, which had greater circulating CD8+ 272 memory T cells, would instead have an advantage in the reinfection setting or following vaccination. 273 Future studies are needed to explore this question, and to better understand sex differences in MAIT cells both in general and in COVID-19. 


\section{MATERIALS AND METHODS}

\section{Ethics statement}

277 This study and relevant protocols were approved by the Institutional Review Boards of Duke University

278 Health System (DUHS) ?. All procedures were performed in accordance with the Declaration of

279 Helsinki, applicable regulations, and local policies.

\section{Participants in this study}

281 In-patients (hospitalized) and out-patients (infected) with confirmed infection of SARS-CoV-2 were

282 identified through the DUHS and enrolled into the Molecular and Epidemiological Study of Suspected 283 Infection (MESSI, Pro00100241). The RT-PCR testing for SARS-CoV-2 was performed at either the

284 North Carolina State Laboratory of Public Health or at clinical laboratories of the DUHS. The exposed 285 group, who closely contacted with COVID-19 patients, presented negative PCR test and negative 286 serology test during longitudinally sampling from the first visit to at least 2 months after, typically 0,7 , 287 14, and 28 days relative to enrollment. Initial severity scores of individuals were recorded through a 288 self-reporting survey on 38 defined symptoms related to COVID-19 plus "other" when enrolled. The exposed group (average score $=9.71$ ) showed a lower severity scores compared with infected (out-

290 patients) group (average score $=18.16$ ). The hospitalized patients presented severe disease symptoms

291 with breath difficulty, cough, fever or chest pain when enrolled, and $77.8 \%$ of them for this study

292 required intensive care unit (ICU) care. All COVID-19 patients were also longitudinally sampled with

293 serology test from enrollment to convalescent phase. Healthy donors were enrolled in 2019 (Duke IRB

294 Protocol Pro00009459) with no diagnosis or symptoms consistent with COVID-19 or other respiratory

295 illness. Written informed consent was obtained from all subjects or legally authorized representatives.

296 Patient Demographics are summarized in Table 1. 
PBMC cells were prepared using Ficoll-Hypaque density gradient method. Briefly, peripheral whole blood was collected in EDTA vacutainer tubes and processed within 8 hours. Blood was diluted 1:2 in PBS then layered onto the Ficoll-Hypaque in $50 \mathrm{ml}$ conical tube and centrifuged at $420 \mathrm{~g}$ for $25 \mathrm{~min}$.

301 Buffy coat was collected and washed with D-PBS by centrifugation at $400 \mathrm{~g}$ for $10 \mathrm{~min}$. Cell pellets were 302 resuspended in D-PBS and washed again. PBMCs were assessed for viability and cell count using Vi303 Cell automated cell counter (Beckman-Coulter). PBMCs were adjusted to $10 \times 10^{6} \mathrm{cells} / \mathrm{ml}$ in 304 cryopreservation media (90\% FBS, 10\% DMSO) and aliquoted into cryopreservation vials on ice. Cells 305 underwent controlled freezing at $-80^{\circ} \mathrm{C}$ using CoolCell LX (BioCision) for $12-24$ hours, then transferred 306 to liquid nitrogen vapor phase.

307 Sample processing for flow cytometry and single cell RNA-sequencing (scRNA-seq)

308 Counts and cell viability of thawed PBMCs were measured by Countess II after a wash with DMEM $30910 \%$ FBS. The cell viability of hospitalized patients ranged from $70-80 \%$ whereas all other samples 310 exceeded $80 \%$ viability. An additional dead cell removal step (Miltenyi Biotec) was conducted on 311 hospitalized PBMC samples prior to aliquot for scRNA-seq. To perform scRNA-seq, 200,000 cells per 312 sample were aliquoted, spun down, resuspended in $30 \mu \mathrm{l}$ PBS supplemented with $0.04 \%$ BSA and $3130.2 \mathrm{U} / \mu \mathrm{l}$ RNase inhibitor and counted using Countess II.

\section{Panel and Staining for Flow Cytometry}

315 Approximately $0.5-2 \times 10^{6}$ cells per cryopreserved sample were stained for flow cytometry analysis.

316 Antibody titrations used in this study were previously established by Cytek Biosciences with slight 317 modifications (see Table S2 for flow panel information). All staining procedures were performed at room 318 temperature. PBMCs were stained with live/dead Blue (Thermofisher) for 15 min, washed with FACS319 EDTA buffer and spun down at 1500 rpm for $5 \mathrm{~min}$. Samples were resuspended with Brilliant Stain 320 Buffer Plus (BD Biosciences) and sequentially stained with anti-CCR7 for 10 min, the chemokine 321 receptor mix for $5 \mathrm{~min}$, anti-TCR gamma/delta for $10 \mathrm{~min}$ and the surface receptor $\mathrm{mix}$ for $30 \mathrm{~min}$. After 
322 incubation, PBMCs were washed with FACS-EDTA buffer and spun down at 1500 rpm for $5 \mathrm{~min}$.

323 Samples were fixed with 1\% PFA in PBS for 20 min, spun down and resuspended in FACS-EDTA

324 buffer.

325 36-color Full Spectrum Flow Cytometry

326 Samples were acquired using a four-laser Cytek Aurora Spectral Flow Cytometry System. Single color

327 controls for spectral unmixing were done with PBMCs from healthy control blood and UltraComp

328 eBeads (ThermoFisher). Raw data were unmixed and further analyzed using either FlowJo for manual

329 gating or Omiq (https://www.omiq.ai) for clustering visualization and analysis.

330 High-dimensional data analysis of flow cytometry data

331 Uniform Manifold Approximation and Projection (UMAP) and FlowSOM clustering analyses were

332 performed on Omiq (https://www.omiq.ai), using equal random sampling of 3000 live CD45+ singlets.

333 from each FCS file. The UMAP plot was generated with the parameters of 15 neighbors and 0.4

334 minimum distance. All markers in flow panel were used for analysis except live/dead and CD45.

335 ScRNA-seq using 10x Genomics platform

336 10x Genomics Single Cell 5' v1 chemistry was used to generate Gel Bead-In Emulsions (GEM), and

337 perform post GEM-RT cleanup, cDNA amplification, as well as library construction. An agilent DNA

338 ScreenTape assay was used for quality control. Libraries were pooled and sequenced to saturation or

33920,000 unique reads per cell on average using an Illumina NovaSeq6000 with 150-bp paired-end

340 reads.

341 Processing and quality control of scRNA-seq

342 Raw sequencing data were initially processed with 10x Genomics Cell Ranger pipelines (V3.1.0).

343 Briefly, BCL files were demultiplexed to generate FASTQ files. FASTQ files were aligned with STAR

344 aligner to the human genome reference GRCh38 from Ensemble database. Feature barcode 
345 processing and UMI counting were then performed according to the standard workflow. (QC summary

346 after sequencing). The following criteria were applied as quality control of single cells from all individual

347 samples. Cells that had fewer than 1000 UMI counts or 500 genes, as well as cell that had greater than

$34810 \%$ of mitochondrial genes were removed from further analysis. Genes that were expressed by fewer

349 than 10 cells were also excluded. After filtering, a total of 424,080 cells with 18,765 gene features were

350 kept for the downstream analysis.

351 Dimensionality reduction and clustering analysis

352 The filtered gene-barcode matrix was analyzed using Seurat 3 (28). All the procedures were conducted

353 with the default parameters unless otherwise specified. Briefly, data were first normalized using log

354 transformation and adjusted with a scale factor of 10,000. The top 2,000 variable genes were identified, 355 and percentages of mitochondrial genes were regressed out when scaling data. Principle component 356 analysis (PCA) was performed using these top variable genes, and top 25 principle components (PCs)

357 were selected for graph-based clustering with Shared Nearest Neighbor (SNN) and visualization in

358 UMAP. The resolution was set to 0.35 to identify major immune cell subsets in PBMCs. Sub-clustering 359 of CD161 hi T cells (21,610 cells) was also performed using the analytic pipeline mentioned above with 360 two modifications: top 10 PCs were used, and the resolution was set to 0.1 to identify MAIT cell 361 clusters.

Differential gene expression analysis

363 Differentially expressed genes (DEGs) were identified using Seurat 3 (FindAllMarkers or FindMarkers 364 Functions) with either 'wilcox' for all cluster markers or 'DESeq2' (47). Randomly downsampled data 365 with 100,000 cells were used to find all markers of PBMC clusters. A gene was considered significant 366 with adjusted $p$-value or false discovery rate $(F D R)<0.05$. DEGs results of all PBMCs and MAIT cells 367 are listed in Table S3 and S4. 
369 Top 100 DEGs of MAIT clusters were used for pathway enrichment analysis using Reactome Pathway

370 Database (https://reactome.org). A pathway was considered significantly over-presented with FDR

$371<0.05$. The full pathway enrichment results are summarized in Table S5.

372 Inference of ligand-receptor interactions between T cells and monocytes

373 Ligand-receptor interactions between T cells and monocytes were inferred using CellPhoneDB (33).

374 PBMC scRNA-seq data were randomly downsampled to 50,000 cells and T and monocyte clusters

375 were extracted based on the expression of their lineage markers. CellPhoneDB was with default

376 parameters (https://github.com/Teichlab/cellphonedb). The inferred interactions are considered

377 significant when $p$-value $<0.05$.

378 Integration of BALF and NPS dataset

379 Publicly available scRNA-seq data of BALF (36) and of NPS (37) were downloaded and processed

380 using Seurat 3 as previously described (28). All T cell clusters, were extracted from both dataset and

381 integrated via Single Cell Transform (SCT) method in Seurat 3. Top 3,000 variable features were

382 selected for the integration. Dimensionality reduction was conducted using PCA and UMAP embedding

383 of the top 100 PCs. Clusters were visualized at a resolution of 0.8 after constructing a SNN graph using 384 the first 50 PCs.

385 Calculations of the feature scores in MAIT cells

386 The DEGs between MAIT1 and MAIT2 were used to generate their feature scores as previously

387 described (48). The feature scores were calculated using AddModuleScore function in Seurat 3. MAIT

388 cells from different single cell dataset were plotted with MAIT1 feature and MAIT2 feature for

389 visualization. 
391 Data normality and homogeneity of variance were assessed using Kolmogorov-Smirnov test and

392 Bartlett's test, respectively. Due to the distribution and variance of human data, non-parametric

393 statistical tests were favorably used throughout this study unless otherwise specified. Mann Whitney $U$

394 test was used for two-group comparisons, and Kruskal-Wallis with post hoc Dunn's test was used for

395 comparisons of three groups and more. Spearman's correlation efficiency was used to quantify the

396 correlation of the ranked disease severity (from healthy as 1 , to hospitalized as 4 ). To adjust $p$-values

397 for multiple hypothesis testing, FDR correction was performed using the Benjamini-Hochberg procedure

398 when appropriate. Two-tailed tests were used unless otherwise specified. A p-value or FDR $<0.05$ is

399 consider statistically significant. Graphical data of quantifications presented throughout are expressed

400 as the means \pm SEMs and were plotted using Graphpad Prism 8. Other graphs in this study were

401 generated using either the corresponding analytic packages or R package ggplot2.

402 Data availability

403 All clinical metadata of participants and samples in this study are included in Table S1. Data will be

404 shared upon the acceptance of this manuscript. Publicly available scRNA-seq data of BALF (36) were

405 downloaded from GEO with the accession number GSE145926, and the count data of NPS (37) were

406 downloaded from https://doi.org/10.6084/m9.figshare.12436517. All of the raw fcs files and all scripts

407 used for data analysis are available to share per request.

409 Acknowledgements: We would like to thank Monica DeLay and Patrick Duncker (Cytek Biosciences)

410 for their help with spectral flow cytometry, and Chris Ciccolella and Geoff Kraker (Omiq, Inc). We would

411 also like to thank Maria Miggs, Deborah Murray, Tyffany Locklear, Robert Rolfe, Jack Anderson, Allison

412 Fullenkamp, Raul Louzuo, Thad Gurley and Julie Steinbrink for their work, as well as the support from

413 Durham Veterans Affairs Health Care System and Duke Regional Hospital. 
414 Funding: This work was supported by NIH/NIAID (U01AI066569, UM1Al104681), the U.S. Defense

415 Advanced Projects Agency (DARPA, N66001-09-C-2082 and HR0011-17-2-0069), the Veterans Affairs

416 Health System, and Virology Quality Assurance (VQA) 75N93019C00015. COVID-19 samples were

417 processed under Biosafety level (BSL)-2 with aerosol management enhancement or BSL-3 in the Duke

418 Regional Biocontainment Laboratory which received partial support for construction from NIH/NIAID

419 (UC6AI058607).

420 Competing interests: MTM reports grants on biomarker diagnostics from the Defense Advanced

421 Research Projects Agency (DARPA), National Institutes of Health (NIH), Sanofi, and the Department of 422 Veterans Affairs. TWB reports grants from DARPA and is a consultant for Predigen; MTM, TWB, ELT, 423 GSG, and CWW report patents pending on Molecular Methods to Diagnose and Treat Respiratory 424 Infections. ELT reports grants on biomarker diagnostics from DARPA, the NIH/Antibacterial Resistance 425 Leadership Group (ARLG) ; an ownership stake in Predigen; GSG reports an ownership stake in 426 Predigen; CWW reports grants on biomarker diagnostics from DARPA, NIH/ARLG, Predigen, and 427 Sanofi; and has received consultancy fees from bioMerieux, Roche, Biofire, Giner, and Biomeme. 


\section{REFERENCES}

429 1. E. Dong, H. Du, L. Gardner, An interactive web-based dashboard to track COVID-19 in real 430 time. Lancet Infect Dis 20, 533-534 (2020).

431 2. E. P. Scully, J. Haverfield, R. L. Ursin, C. Tannenbaum, S. L. Klein, Considering how biological sex impacts immune responses and COVID-19 outcomes. Nat Rev Immunol 20, 442-447 (2020).

$4343 . \quad$ I. G. Alghamdi et al., The pattern of Middle East respiratory syndrome coronavirus in Saudi

4. J. Karlberg, D. S. Chong, W. Y. Lai, Do men have a higher case fatality rate of severe acute

5. H. N. Leong et al., SARS in Singapore--predictors of disease severity. Ann Acad Med Singap Arabia: a descriptive epidemiological analysis of data from the Saudi Ministry of Health. Int J

6. R. Channappanavar et al., Sex-Based Differences in Susceptibility to Severe Acute Respiratory Syndrome Coronavirus Infection. J Immunol 198, 4046-4053 (2017).

7. S. L. Klein, K. L. Flanagan, Sex differences in immune responses. Nat Rev Immunol 16, 626-

8. M. J. Polanczyk et al., Cutting edge: estrogen drives expansion of the CD4+CD25+ regulatory T

9. D. Furman et al., Systems analysis of sex differences reveals an immunosuppressive role for testosterone in the response to influenza vaccination. Proc Natl Acad Sci U S A 111, 869-874 (2014).

10. M. Griesbeck et al., Sex Differences in Plasmacytoid Dendritic Cell Levels of IRF5 Drive Higher

11. D. Mathew et al., Deep immune profiling of COVID-19 patients reveals distinct immunotypes

12. A. Silvin et al., Elevated Calprotectin and Abnormal Myeloid Cell Subsets Discriminate Severe

13. J. Schulte-Schrepping et al., Severe COVID-19 Is Marked by a Dysregulated Myeloid Cell

14. E. R. Mann et al., Longitudinal immune profiling reveals key myeloid signatures associated with

15. Y. Peng et al., Broad and strong memory CD4(+) and CD8(+) T cells induced by SARS-CoV-2

16. T. Sekine et al., Robust T Cell Immunity in Convalescent Individuals with Asymptomatic or Mild COVID-19. Cell 183, 158-168 e114 (2020). 
464 17. N. Le Bert et al., SARS-CoV-2-specific T cell immunity in cases of COVID-19 and SARS, and

18. D. Weiskopf et al., Phenotype and kinetics of SARS-CoV-2-specific T cells in COVID-19

19. D. M. Del Valle et al., An inflammatory cytokine signature predicts COVID-19 severity and

20. T. Takahashi et al., Sex differences in immune responses that underlie COVID-19 disease outcomes. Nature, (2020).

21. M. T. McClain et al., Dysregulated transcriptional responses to SARS-CoV-2 in the periphery support novel diagnostic approaches. medRxiv, (2020).

22. E. Becht et al., Dimensionality reduction for visualizing single-cell data using UMAP. Nat Biotechnol, (2018).

23. S. Van Gassen et al., FlowSOM: Using self-organizing maps for visualization and interpretation

24. Z. Chen, E. John Wherry, T cell responses in patients with COVID-19. Nat Rev Immuno/ 20,

25. T. Parrot et al., MAIT cell activation and dynamics associated with COVID-19 disease severity.

26. L. Kuri-Cervantes et al., Comprehensive mapping of immune perturbations associated with severe COVID-19. Sci Immunol 5, (2020).

27. Y. Jouan et al., Phenotypical and functional alteration of unconventional T cells in severe COVID-19 patients. J Exp Med 217, (2020).

28. T. Stuart et al., Comprehensive Integration of Single-Cell Data. Cell 177, 1888-1902 e1821 (2019).

29. G. Chen et al., Clinical and immunological features of severe and moderate coronavirus disease 2019. J Clin Invest 130, 2620-2629 (2020).

30. Q. Zhao et al., Lymphopenia is associated with severe coronavirus disease 2019 (COVID-19) infections: A systemic review and meta-analysis. Int $J$ Infect Dis 96, 131-135 (2020).

31. L. Tan et al., Lymphopenia predicts disease severity of COVID-19: a descriptive and predictive study. Signal Transduct Target Ther 5, 33 (2020).

32. C. Huang et al., Clinical features of patients infected with 2019 novel coronavirus in Wuhan,

33. M. Efremova, M. Vento-Tormo, S. A. Teichmann, R. Vento-Tormo, CellPhoneDB: inferring cellcell communication from combined expression of multi-subunit ligand-receptor complexes. Nat Protoc 15, 1484-1506 (2020). 
34. A. J. Wilk et al., A single-cell atlas of the peripheral immune response in patients with severe COVID-19. Nat Med 26, 1070-1076 (2020).

35. H. Flament et al., Outcome of SARS-CoV-2 infection linked to MAIT cell activation and cytotoxicity: evidence for an IL-18 dependent mechanism. medRxiv, (2020).

36. M. Liao et al., Single-cell landscape of bronchoalveolar immune cells in patients with COVID-19. Nat Med 26, 842-844 (2020).

37. R. L. Chua et al., COVID-19 severity correlates with airway epithelium-immune cell interactions identified by single-cell analysis. Nat Biotechno/ 38, 970-979 (2020).

38. S. De Biasi et al., Marked T cell activation, senescence, exhaustion and skewing towards TH17 in patients with COVID-19 pneumonia. Nat Commun 11, 3434 (2020).

39. M. Merad, J. C. Martin, Pathological inflammation in patients with COVID-19: a key role for monocytes and macrophages. Nat Rev Immuno/ 20, 355-362 (2020).

40. J. Novak, J. Dobrovolny, L. Novakova, T. Kozak, The decrease in number and change in phenotype of mucosal-associated invariant $\mathrm{T}$ cells in the elderly and differences in men and women of reproductive age. Scand J Immunol 80, 271-275 (2014).

41. O. J. Lee et al., Circulating mucosal-associated invariant $T$ cell levels and their cytokine levels in healthy adults. Exp Gerontol 49, 47-54 (2014).

42. P. Chen et al., Circulating Mucosal-Associated Invariant T Cells in a Large Cohort of Healthy Chinese Individuals From Newborn to Elderly. Front Immunol 10, 260 (2019).

43. N. Chen et al., Epidemiological and clinical characteristics of 99 cases of 2019 novel coronavirus pneumonia in Wuhan, China: a descriptive study. Lancet 395, 507-513 (2020).

44. A. G. Laing et al., A dynamic COVID-19 immune signature includes associations with poor prognosis. Nat Med 26, 1623-1635 (2020).

45. S. Gerart et al., Human iNKT and MAIT cells exhibit a PLZF-dependent proapoptotic propensity that is counterbalanced by XIAP. Blood 121, 614-623 (2013).

46. J. Dias, E. Leeansyah, J. K. Sandberg, Multiple layers of heterogeneity and subset diversity in human MAIT cell responses to distinct microorganisms and to innate cytokines. Proc Natl Acad Sci U S A 114, E5434-E5443 (2017).

47. M. I. Love, W. Huber, S. Anders, Moderated estimation of fold change and dispersion for RNAseq data with DESeq2. Genome Biol 15, 550 (2014).

48. I. Tirosh et al., Dissecting the multicellular ecosystem of metastatic melanoma by single-cell RNA-seq. Science 352, 189-196 (2016). 
Table 1. Summary of Patient Demographics and Sample Information

\begin{tabular}{|c|c|c|c|c|c|c|}
\hline & Group & healthy & exposed & infected & hospitalized & total \\
\hline \multicolumn{2}{|c|}{$\begin{array}{l}\text { Age } \\
\text { mean } \pm S D \text { (range) }\end{array}$} & $\begin{array}{c}39.70 \pm 13.68 \\
(25-61)\end{array}$ & $\begin{array}{c}42.57 \pm 13.93 \\
(17-60)\end{array}$ & $\begin{array}{c}36.73 \pm 13.88 \\
(20-65)\end{array}$ & $\begin{array}{c}59.44 \pm 15.11 \\
(31-76)\end{array}$ & $\begin{array}{c}42.84 \pm 16.06 \\
(17-76)\end{array}$ \\
\hline \multicolumn{2}{|c|}{$\begin{array}{l}\text { \# subjects } \\
\text { (F:M ratio) }\end{array}$} & $\begin{array}{c}n=10 \\
(5: 5)\end{array}$ & $\begin{array}{l}n=7 \\
(3: 4)\end{array}$ & $\begin{array}{l}\mathrm{n}=19 \\
(8: 11)\end{array}$ & $\begin{array}{l}n=9 \\
(4: 5)\end{array}$ & $\begin{array}{l}n=45 \\
(20: 25)\end{array}$ \\
\hline \multirow{4}{*}{$\begin{array}{l}\text { Race } \\
\mathrm{n}(\%)\end{array}$} & $\begin{array}{l}\text { African } \\
\text { American }\end{array}$ & $3(30.00 \%)$ & 0 & $1(5.26 \%)$ & $5(55.56 \%)$ & $9(20.00 \%)$ \\
\hline & Asian & 0 & $1(14.29 \%)$ & 2 (10.53\%) & 0 & $3(6.67 \%)$ \\
\hline & White & 7 (70.00\%) & $5(71.42 \%)$ & $16(84.21 \%)$ & 3 (33.33\%) & $31(68.89 \%)$ \\
\hline & $\begin{array}{l}\text { Others/ } \\
\text { unknown }\end{array}$ & 0 & $1(14.29 \%)$ & 0 & $1(11.11 \%)$ & $2(4.44 \%)$ \\
\hline \multicolumn{2}{|c|}{$\begin{array}{l}\text { Days since onset } \\
\text { when enrolled } \\
\text { mean } \pm S D \text { (range) }\end{array}$} & $\mathrm{N} / \mathrm{A}$ & $\begin{array}{c}15.17 \pm 11.25 \\
(5-33)\end{array}$ & $\begin{array}{c}11.18 \pm 4.30 \\
(3-19)\end{array}$ & $\begin{array}{c}8.25 \pm 6.14 \\
\quad(1-18)\end{array}$ & $\begin{array}{c}11.19 \pm 6.73 \\
(1-33)\end{array}$ \\
\hline \multicolumn{2}{|c|}{$\begin{array}{l}\text { \# samples of flow } \\
\text { cytometry (F:M ratio) }\end{array}$} & $\begin{array}{c}n=10 \\
(5: 5)\end{array}$ & $\begin{array}{l}n=20 \\
(9: 11)\end{array}$ & $\begin{array}{l}n=44 \\
(21: 23)\end{array}$ & $\begin{array}{l}n=9 \\
(4: 5)\end{array}$ & $\begin{array}{l}n=83 \\
(39: 44)\end{array}$ \\
\hline \multicolumn{2}{|c|}{$\begin{array}{l}\text { \# samples of scRNA- } \\
\text { seq (F:M ratio) }\end{array}$} & $\begin{array}{c}n=5 \\
(F: M=3: 2)\end{array}$ & $\begin{array}{c}n=8 \\
(F: M=3: 5)\end{array}$ & $\begin{aligned} n & =29 \\
(F: M & =12: 17)\end{aligned}$ & $\begin{array}{c}n=6 \\
(F: M=1: 5)\end{array}$ & $\begin{aligned} n & =48 \\
(F: M & =19: 29)\end{aligned}$ \\
\hline \multicolumn{2}{|c|}{$\begin{array}{l}\text { Days since onset } \\
\text { when samples collected } \\
\text { mean } \pm S D \text { (range) }\end{array}$} & $N / A$ & $\begin{array}{c}26.94 \pm 14.94 \\
(5-61)\end{array}$ & $\begin{array}{c}18.27 \pm 8.44 \\
(3-40)\end{array}$ & $\begin{array}{c}8.25 \pm 6.14 \\
\quad(1-18)\end{array}$ & $\begin{array}{c}19.32 \pm 11.44 \\
(1-61)\end{array}$ \\
\hline
\end{tabular}


Fig. 1 Sex-Specific CD8+ ${ }^{+}$cell Responses in PBMCs of COVID-19 Patients. (A) Overview of patient groups in this study. (B) UMAP visualization of PBMC subsets identified by FlowSOM clustering. Samples from all participants were pooled and down-sampled to 3,000 live CD45+ cells per sample. MO, monocytes; NK, natural killer cells; DC, dendritic cells, PMNs, polymorphonuclear neutrophils. Baso, basophils. (C) Expression of CD161, TCR yס and CD56 in CD8+ T cell subsets. (D) UMAP of samples grouped by disease severity. Samples collected within 3 days from enrollment were included. (E) Frequencies of CD161 ${ }^{\text {hi }} \mathrm{T}$ cells in different severity groups (left) and their correlation with severity rank (right). (F) UMAP of samples stratified by sex and time post symptom onset (early, $\leq 14$ days; middle, $>15$ days and $\leq 21$ days; late, $>22$ days). (G) Frequencies of CD161 ${ }^{\text {hi }}$ and memory CD8 ${ }^{+} T$ cells between sex and timepoints. (H) Sex-specific changes of CD161 ${ }^{\text {hi }}$ cells frequencies shown in $\mathrm{G}$.

(I) Frequencies of CD161 $1^{\mathrm{hi}}$ and memory CD8 ${ }^{+} \mathrm{T}$ cells in the samples from confirmed COVID-19 subjects pre- and post-seroconversion. Data were plotted as mean \pm standard error. Significance was determined by Kruskal-Wallis test with Dunn's test $(E, H)$ or Mann Whitney test $(G, I):{ }^{*} p<0.05$, ${ }^{* *} p<0.01$.

Fig. 2 Characterization of CD8 ${ }^{+}$CD161 1 hi $T$ cells in COVID-19 using scRNA-seq. (A) UMAP and unsupervised cluster analysis of PBMCs. MO, monocytes; RBC, red blood cells; PB, plasmablasts; in UMAP (B) and expression of their marker genes as indicated in Violin plots (C). T $^{*}$ cluster likely represents a dropout population with low UMI counts. N, naïve; EM, effect memory; CM, central memory; DN, double negative; rep, replicating. (D) Changes of T cell subsets with disease severity. $\mathrm{N}$, the number of individuals. (E) Frequencies of $\mathrm{CD} 161^{\text {hi }}$ cluster relative to all T cell subsets. Females were plotted in red and males in blue. Red dash box delineated the healthy females. (F) Top enriched pathways of $\mathrm{CD} 161^{\text {hi }}$ cluster in Reactome Pathway Database ranked by false discovery rate (FDR, $\log 10$ scale). Data were plotted as mean \pm standard error. Significance was determined by Kruskal- 
Fig. 3 Heterogeneity and Dynamics of Circulating MAIT cells across Sex in COVID-19. (A) Sub-

561 clustering of CD16 $1^{\text {hi }}$ cells $(n=21,610)$ showing two MAIT clusters and one $ү \delta$ T cluster. (B) Marker

562 gene expression of three CD16 $1^{\text {hi }}$ clusters. (C) Heatmap of top 25 discriminative genes between

563 MAIT $\alpha$ and MAIT $\beta$ clusters. Expression level was scaled by Z-score distribution. (D and E)

564 Representative top enriched pathways of MAIT $\alpha$ and MAIT $\beta$ in Reactome Pathway Database (ranked

565 by false discovery rate, -log10 scale). Top 100 DEGs ranked by fold change between MAIT $\alpha$ and

566 MAIT $\beta$ were used for this analysis. (F and $\mathbf{G})$ UMAP visualization of MAIT cluster changes $(F)$ and their

567 frequencies $(\mathrm{G})$ with disease severity. $(\mathrm{H})$ Frequencies of MAIT clusters grouped by time post symptom

568 onset. (I and $\mathbf{J}$ ) Sex differences of MAIT clusters as shown in H. Data were plotted as mean \pm standard

569 error (G-J). Significance was determined by Mann Whitney test (I). ${ }^{*} \mathrm{p}<0.05$.

570 Fig. 4 MAIT Cell Differences by Sex in Airway Tissue Samples of COVID-19 Patients. (A and B)

571 Clustering analysis of scRNA-seq data from COVID-19 BALF dataset with subtracted T and NK cells

572 (36). (C) MAIT cell cluster indicated by marker genes. (D) Frequencies of MAIT cells in BALF between

573 normal and COVID-19 subjects (left) and across sex within COVID-19 subjects (right). (E) Integrated

574 clustering analysis of NPS with BALF using Seurat 3. (F) Referenced MAIT cluster in NPS by the

575 expression of TRAV1-2 in BALF and indicated marker genes in NPS. (G) Frequencies of MAIT cells in

576 NPS from healthy and COVID-19 subjects. (H and I) Visualization $(H)$ and frequencies (I) of MAIT cells

577 in NPS grouped by disease severity. (J) Volcano plot showing of DEGs of BALF MAIT cells between

578 sex with fold change and FDR. (K to $\mathbf{M}$ ) Expression of DEGs in IL-7 signaling (K), transcriptional factors

579 (L) and CCL2 (M). Data were plotted as mean \pm standard error (D, G, I) with females in red and males

580 in black. Significance was determined by unpaired a one-tailed student's t-test (D), Kruskal-Wallis test

581 with Dunn's post hoc test $(\mathrm{I})$ and Mann Whitney test $(\mathrm{G}):{ }^{*} \mathrm{p}<0.05,{ }^{* *} \mathrm{p}<0.01$. 
Supplementary Materials

583 Table S1. Clinical Metadata of Participants and Samples in this study

Table S2. List of Panel Reagents for 36-color Spectral Flow Cytometry

Table S3. List of DEGs of PBMC Clusters and MAIT cells

Table S4. List of DEGs of MAIT Subsets

Table S5. List of Enriched Reactome Pathways of MAIT Subsets

Figure S1. Annotation of Immune Subsets and Gating Strategy of PBMCs from COVID-19 patients Using 36-color Flow Cytometry. (A) Annotation of subsets for CD4 T, CD8 T cells, үס T cells, monocytes, B cells and NK cells as shown in fig. 1B. Left-hand side in each panel is the UMAP with the indicated immune cell population (box) and the respective subsets annotated by color (legend). Right-hand side is feature plots of the indicated population showing relative expression of each marker (black= negligible; white $=$ positive). $($ B) Gating strategy of immune cell subsets in PBMC samples.

Figure S2. Frequency Profiling of Immune Subsets in COVID-19 PBMCs among Different Disease Severity groups. Gating strategy was shown in Fig. S1B. All data were plotted as percentage of CD45+ PBMCs. Significance was determined by Kruskal-Wallis test.

\section{Figure S3. Sex-Specific Changes of CD8 ${ }^{+}$CD161 ${ }^{\text {hi }}$ and Memory T Cells in COVID-19 PBMCs by}

Flow Cytometry. (A) Gating strategy of CD8+ CD161hi, memory and naive T cells ( $\alpha \beta)$. (B)

Representative flow plots of $\mathrm{CD} 8^{+} \mathrm{CD} 161^{\mathrm{hi}}$ and memory $\mathrm{T}$ cells from female and male individuals with disease severity. (C) Frequencies of naïve CD8 T cells in different severity groups as indicated.

601 Significance was calculated by Kruskal-Wallis. 
602 Figure S4. Characterization of Immune Subsets in COVID-19 PBMCs by scRNA-seq. (A) High

603 resolution clustering of PBMCs shown in Fig.2A. (B) Expression of marker genes used to annotate 604 individual clusters. cl, classical; int, intermediate; nc, non-classical; CD14 MOA, activated CD14 605 monocytes; CD16 MOA, activated CD16 monocytes; PB, plasmablasts; PLT, platelets.

Figure S5. Interaction Inference of Receptors and Ligands between Monocytes and Major T Cell

607 Subsets. (A and B) Visualization (A) and percentage distribution (B) of different monocyte subsets 608 identified in Fig.S4A. Three monocyte subsets represent resting classical CD14 (cl), non-classical 609 CD16 (nc), and intermediate (int) monocytes as seen in healthy subjects. Two monocyte subsets are 610 associated with interferon signaling and COVID-19 patients with differential expression of CD14 and 611 CD16, referred to as activated CD14 and activated CD16 monocytes $\left(C D 14 \mathrm{MO}_{\mathrm{A}}\right.$ and CD16 MOA, 612 respectively). (C) Overview of selected ligand-receptor interactions inferenced by CellPhoneDB in 613 COVID PBMC single cell dataset. Red dash box delineated the specific interaction of CD161 ${ }^{\text {hi }}$ T cells 614 with monocytes. P values and scales are indicated by circle size and colors, respectively. (D) 615 Expression of representative ligand and receptor pairs between MAIT and monocytes as indicated. (E) Heatmap of interaction counts between major T cell and monocyte subsets.

Figure S6. Expression of CD69 by circulating MAIT Clusters in COVID-19. (A to D) Data are grouped by MAIT clusters (A), sex (B), disease severity (C) as well as clusters and severity (D).

Figure S7. Comparisons of MAIT $\alpha$ and MAIT $\beta$ Features in MAIT Cells from peripheral blood and airway tissue samples. (A) Estimation of MAIT $\alpha$ and MAIT $\beta$ features of individual cells from PBMC and BALF dataset based on the expression of MAITa gene (y-axis) and MAIT $\beta$ gene sets ( $x$-axis). Differentially expressed genes between MAIT $\alpha$ and MAIT $\beta$ were used as two modular features, respectively. (B to D) Expression of IL7 receptor and its co-receptor genes (B), apoptosis-related genes (C), proliferation-related genes by MAIT cells from PBMC and BALF dataset (D). 

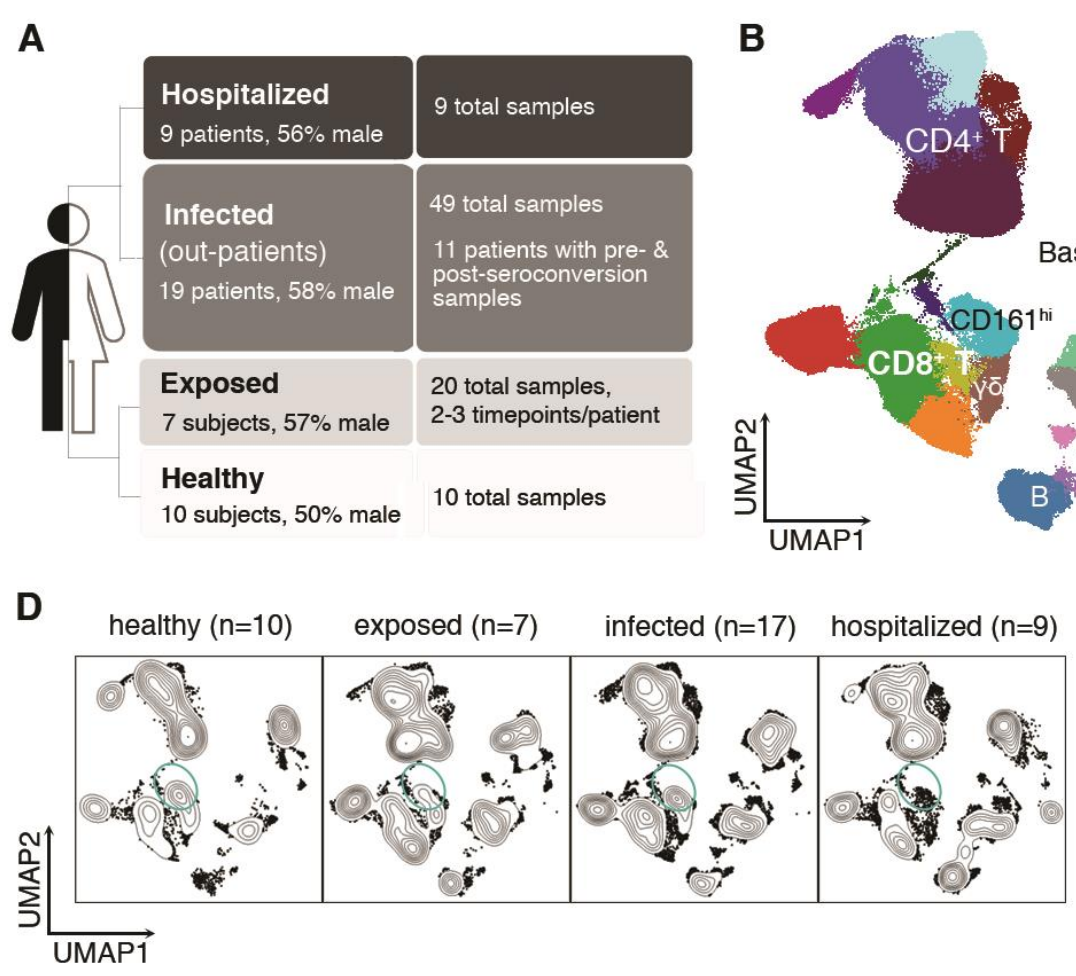

$\mathbf{F}$

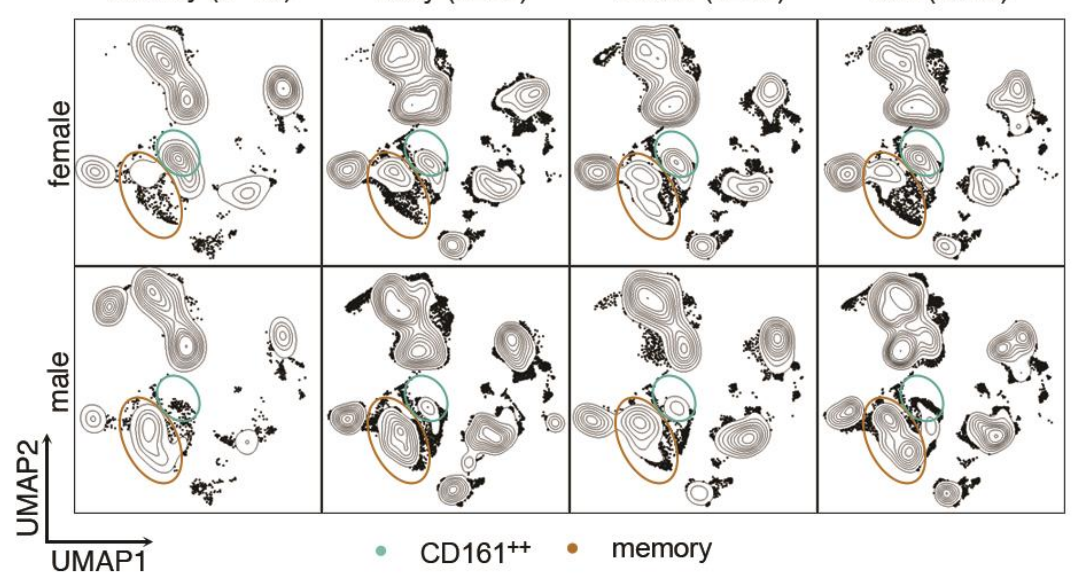

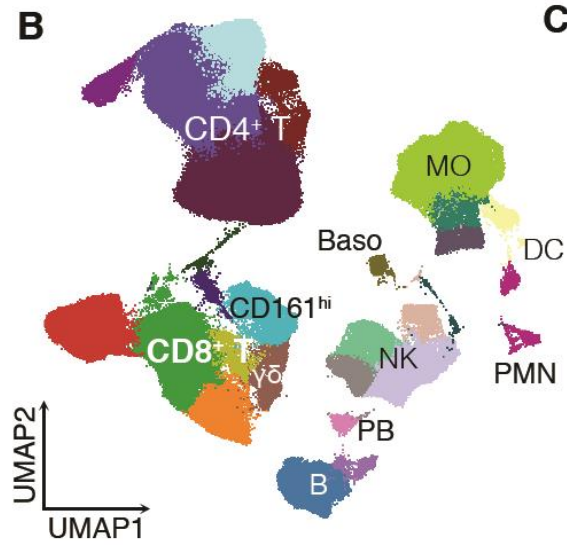

C $\quad$ CD8 ${ }^{+}$T subsets

$E$

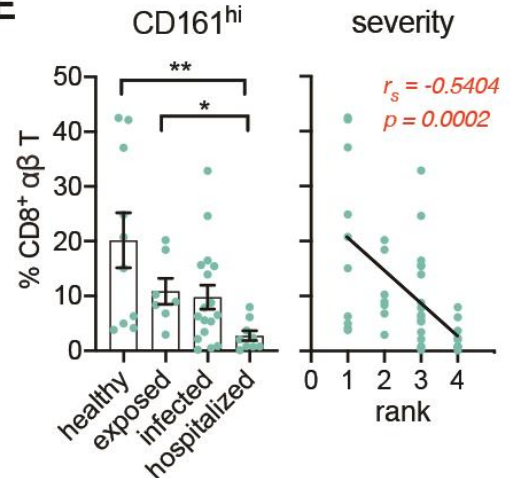

$\mathbf{H}$

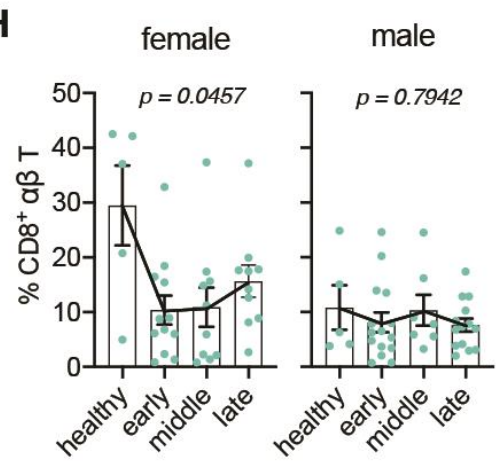

G

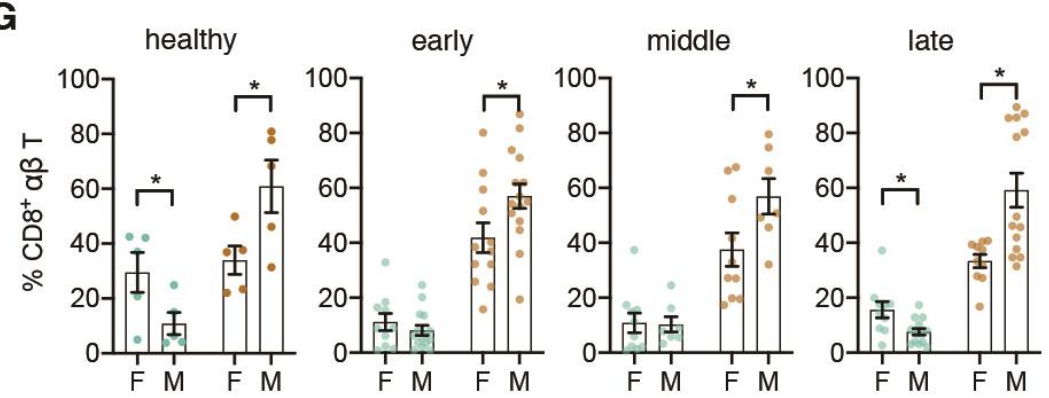

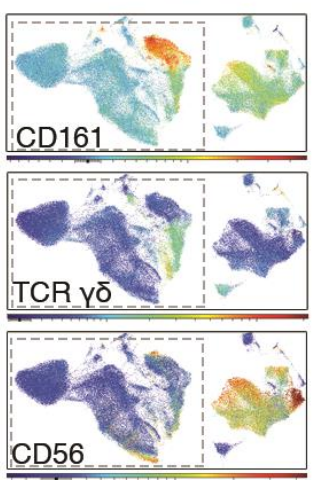

CD161 ${ }^{\text {hi }}$ memory

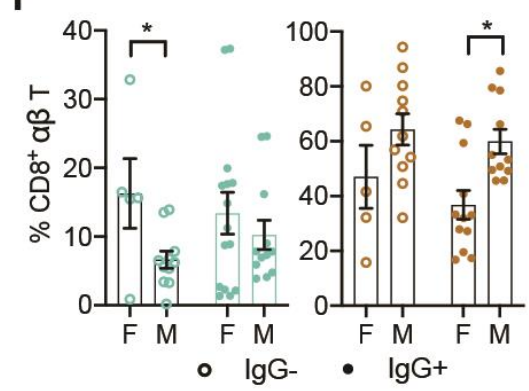


bioRxiv preprint doi: https://doi org/10.1101/2020.12.01.407148; this version posted December 1, 2020. The copyright holder for this preprint (which was not certified by peer review) is the author/funder, who has granted bioRxiv a license to display the preprint in perpetuity. It is made available under aCC-BY-NC-ND 4.0 International license.

\section{Figure 2}

A

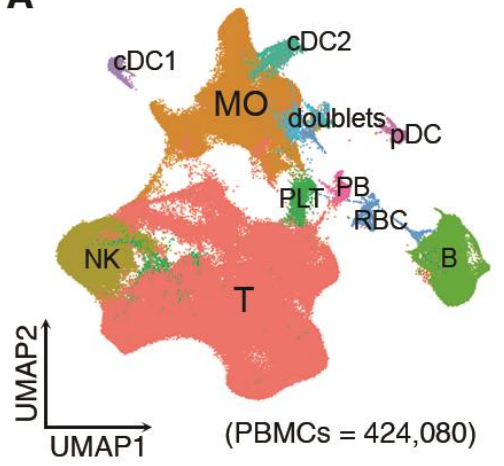

B

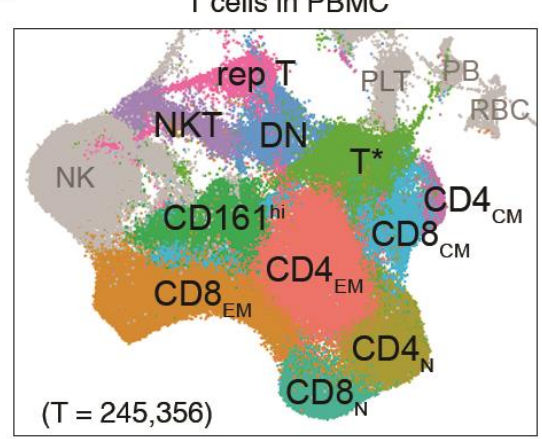

- $\mathrm{CD} 4_{\mathrm{EM}}$ - $C D 8_{E M}$ - $\mathrm{CD}^{*} \mathrm{~N}$ - CD161 ${ }^{\mathrm{hi}}$ - $\mathrm{CD}_{\mathrm{N}}$ - $\mathrm{CD} 8_{\mathrm{CM}}$ - DN

- NKT

- $\mathrm{CD}_{\mathrm{CM}}$

- rep T

D

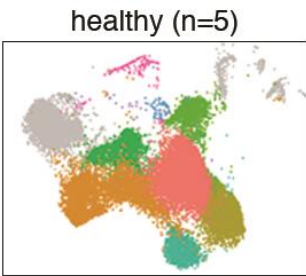

infected $(\mathrm{n}=10)$

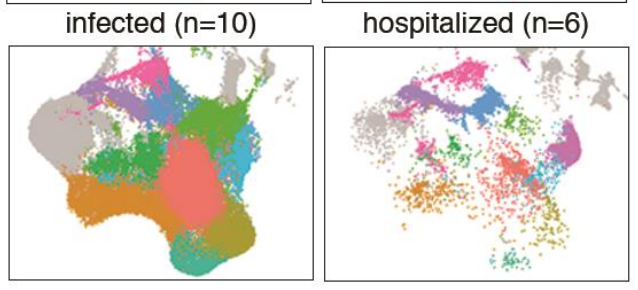

$\mathbf{E}$
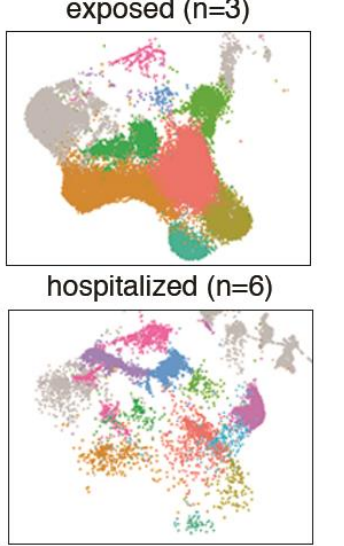

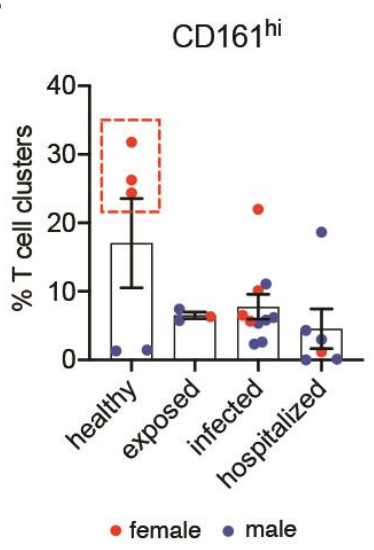

$\mathbf{F}$

NGF-stimulated transcription Response of EIF2AK1 (HRI) to heme deficiency

Nuclear Events (kinase and transcription factor activation)

Signaling by NTRK1 (TRKA)

Signaling by TGF-beta Receptor Complex $\mathrm{NR} 1 \mathrm{H} 2$ and NR1H3mediated signaling IL-10 signaling

RAF-independent MAPK $1 / 3$ activation Estrogen-dependent nuclear events downstream of ESR-
membrane signaling 
bioRxiv preprint doi: https://doi.org/10.1101/2020.12.01.407148; this version posted December 1, 2020. The copyright holder for this preprint (which was not certified by peer review) is the author/funder, who has granted bioRxiv a license to display the preprint in perpetuity. It is made available under aCC-BY-NC-ND 4.0 International license.

\section{$630 \quad$ Figure 3}

A

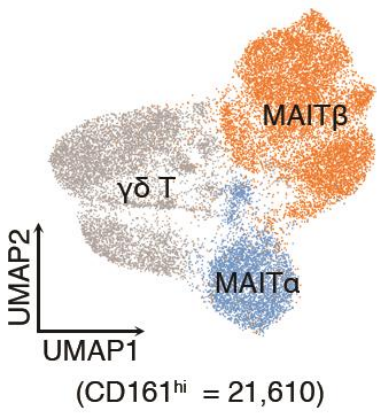

B

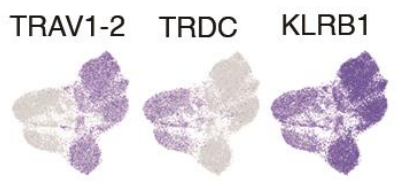

D

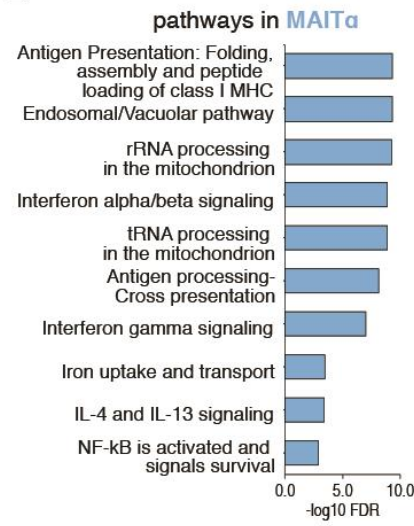

$\mathbf{F}$

\begin{tabular}{|c|c|c|c|}
\hline healthy & exposed & infected & hospitalized \\
\hline & & & $\theta$ \\
\hline & $\therefore$ & & (e) \\
\hline
\end{tabular}

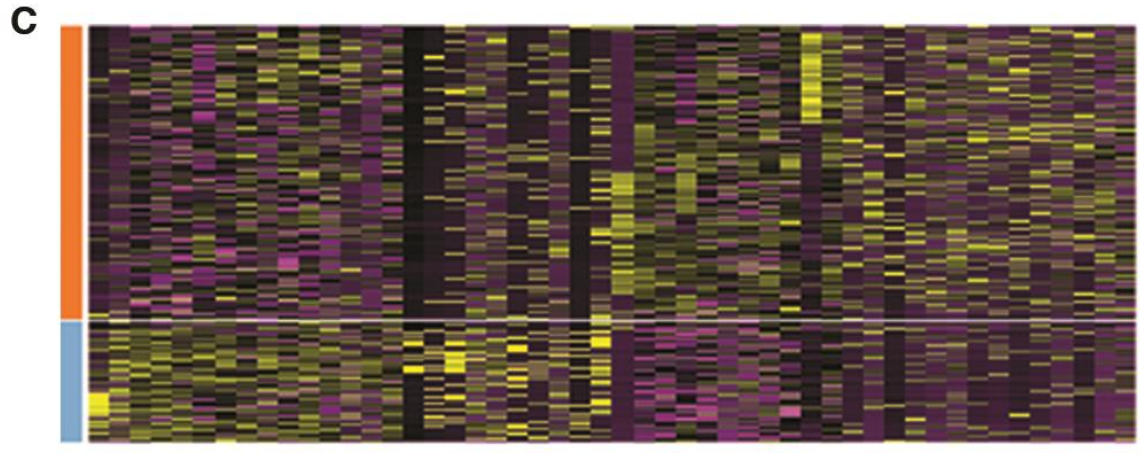

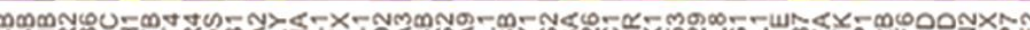

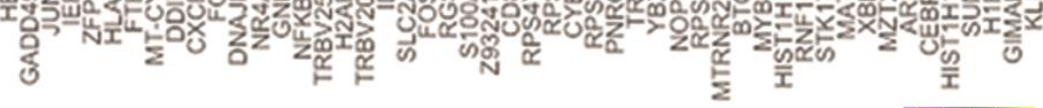

- MAITa - MAIT $\beta$

E

pathways in MAIT $\beta$

Formation of the ternary

ranslation initiation complex formation

Peptide chain elongation

Viral mRNA Translation

Influenza Infection

Cellular responses to stress Apoptosis induced

Unfolded Protein Response

Apoptotic execution phase

DNA Damage/Telomere Stress Induced Senescence

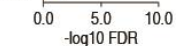

G

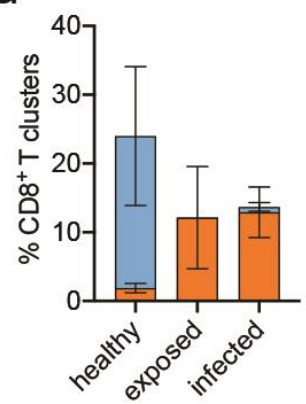

$\mathbf{H}$

I

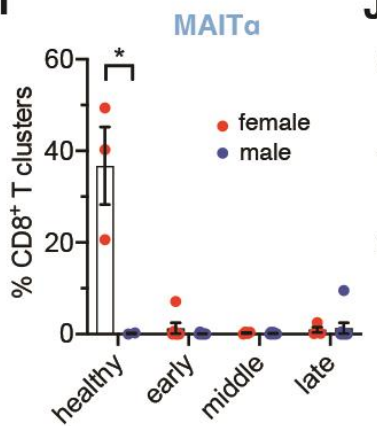

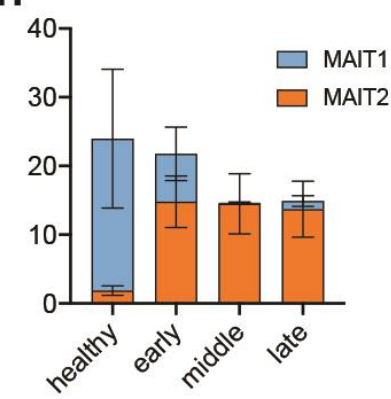

J

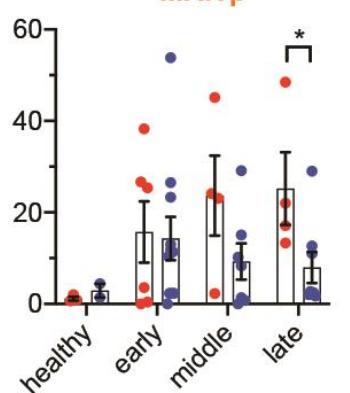


bioRxiv preprint doi: https://doi.org/10.1101/2020.12.01.407148; this version posted December 1, 2020. The copyright holder for this preprint (which was not certified by peer review) is the author/funder, who has granted bioRxiv a license to display the preprint in perpetuity. It is made available under aCC-BY-NC-ND 4.0 International license.

\section{Figure 4}

A

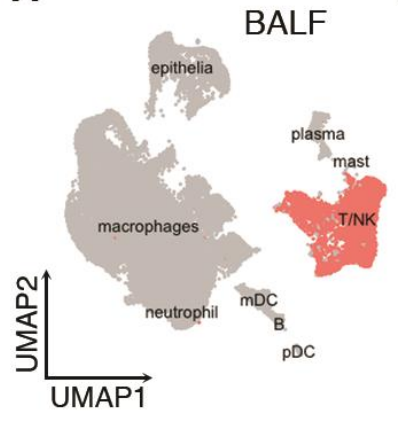

B

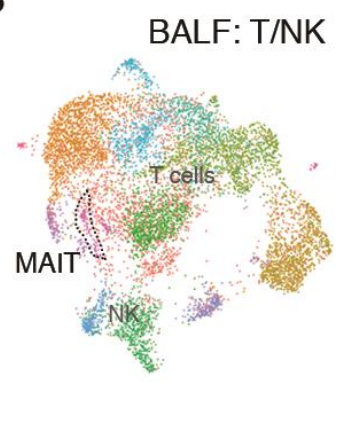

C

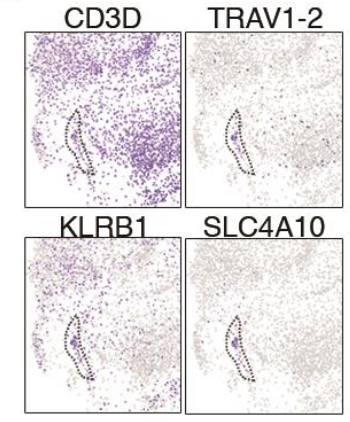

D MAIT in BALF

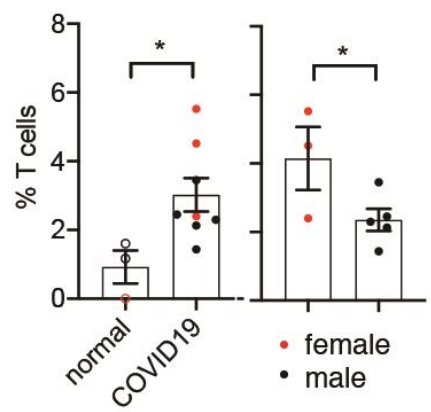

E

BALF: T

NPS: T
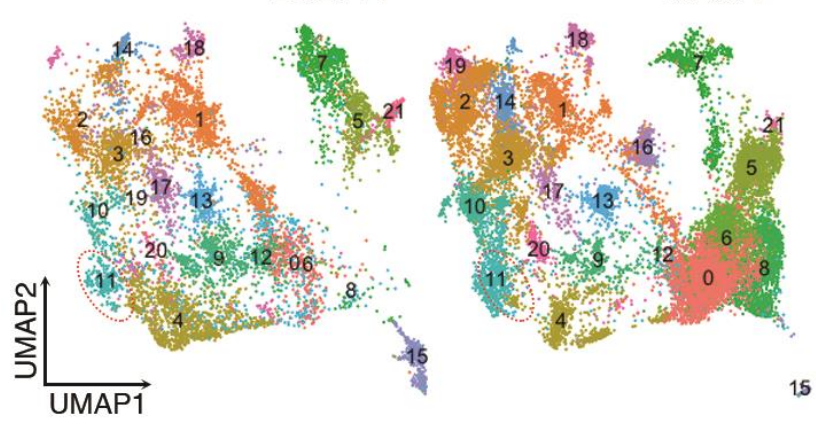

F

H
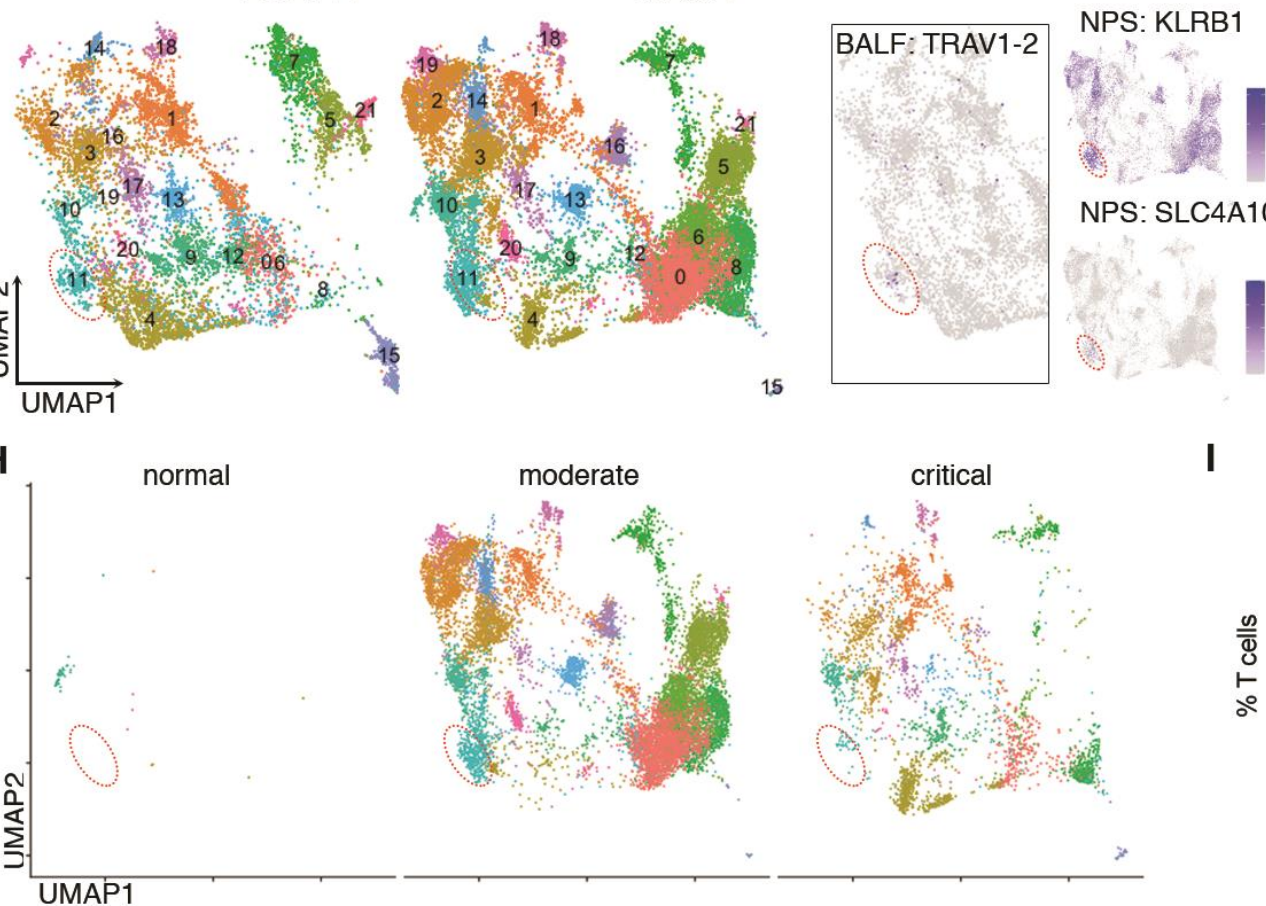

NPS: SLC4A10

G MAIT in NPS

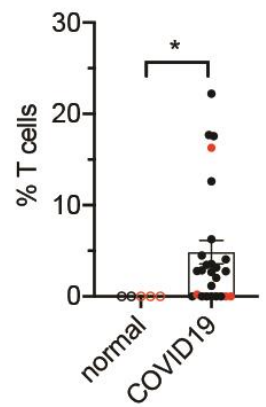

MAIT in NPS
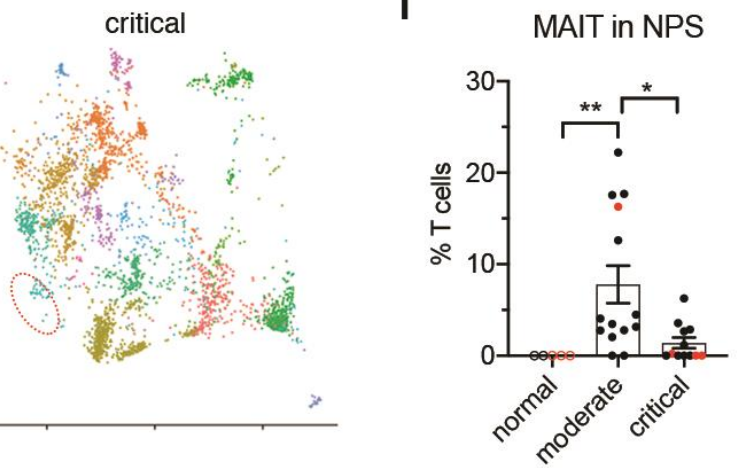

J RPS4Y1

MTRNR2L12•

TXNiP

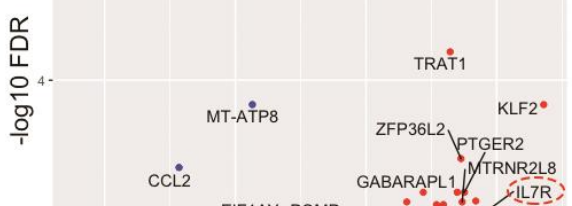

EIF1AY POMP GABARAPL11 GIILZ CXCL10 ITM2A ' TMSB4Xั. "CEBPD

- CD74 \%

$\therefore$ ¡ं

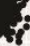

K

K IL7R
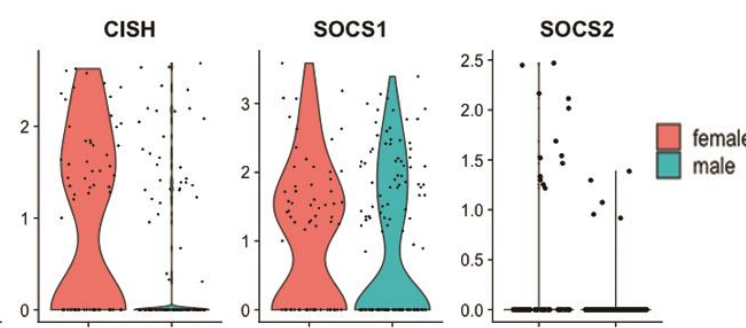

L
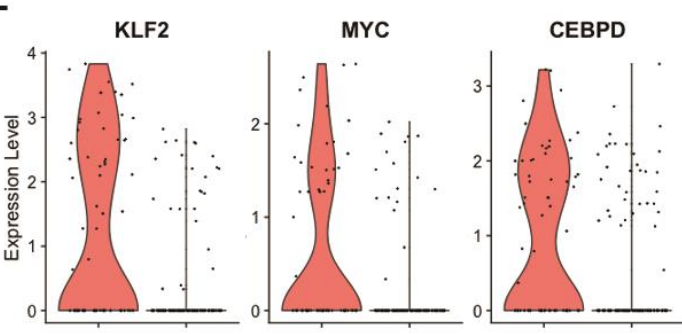

M

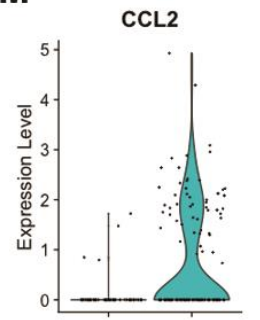


bioRxiv preprint doi: https://doi.org/10.1101/2020.12 01.407148; this version posted December 1, 2020. The copyright holder for this preprint (which was not certified by peer review) is the author/funder, who has granted bioRxiv a license to display the preprint in perpetuity. It is made available under aCC-BY-NC-ND 4.0 International license.

\section{Supplementary Figure S1}

A
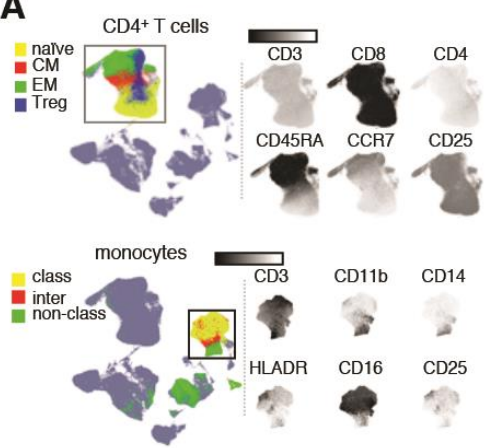
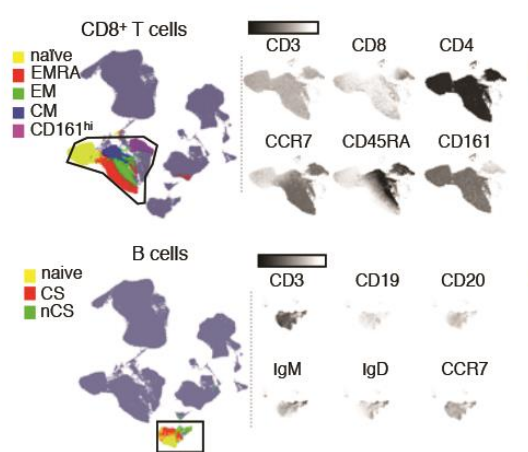

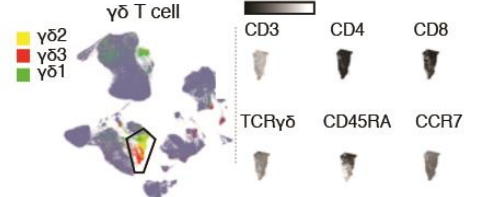

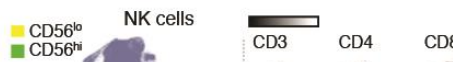

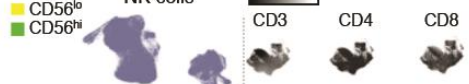

$\therefore$ CD56 CD45RA CD16
B
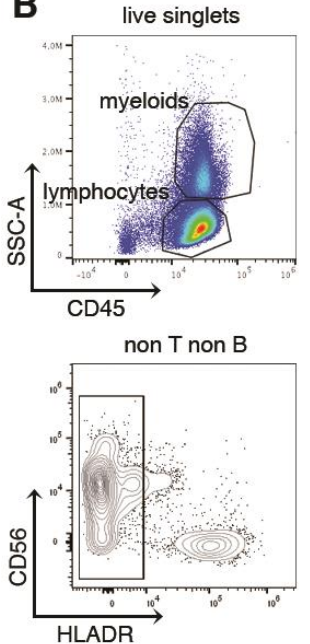

C
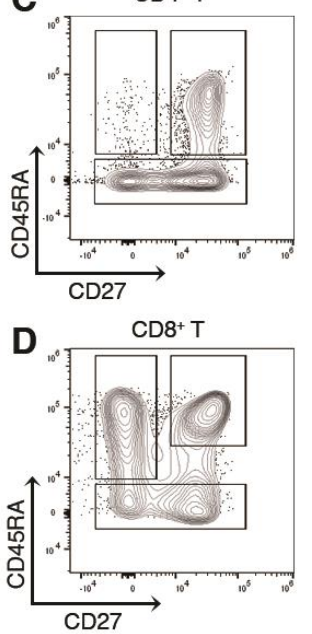

E

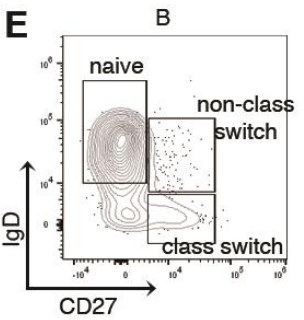

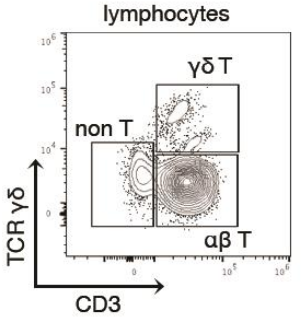
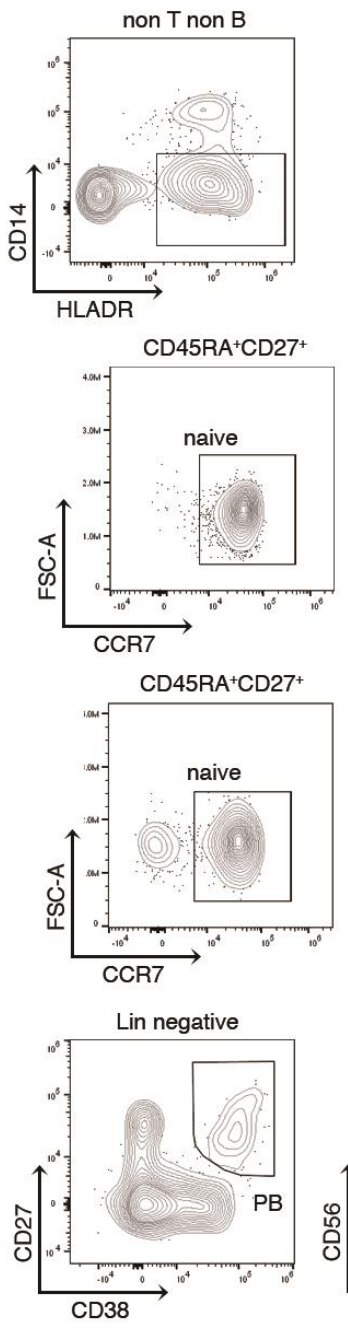
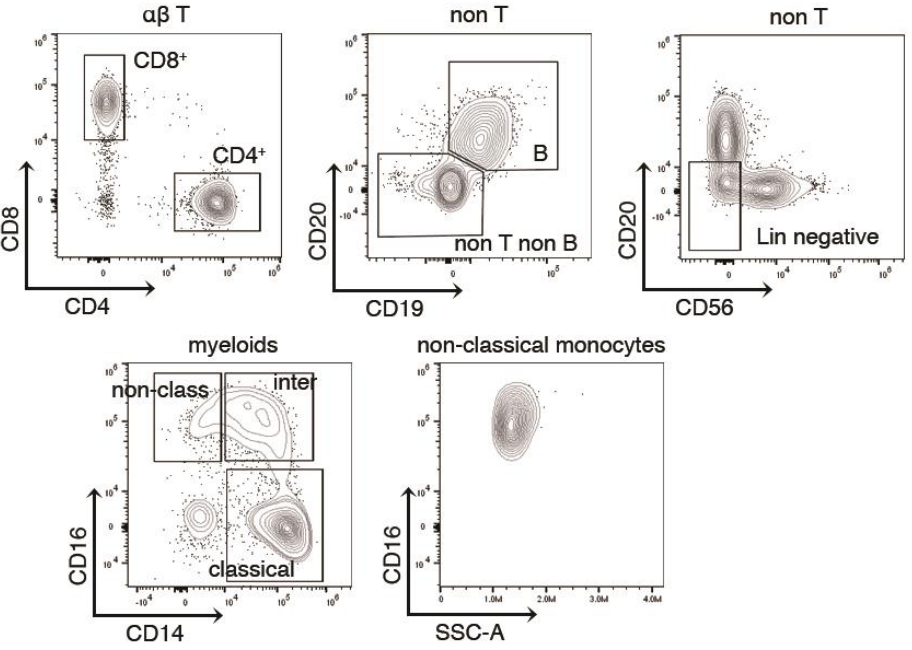

non-classical monocytes
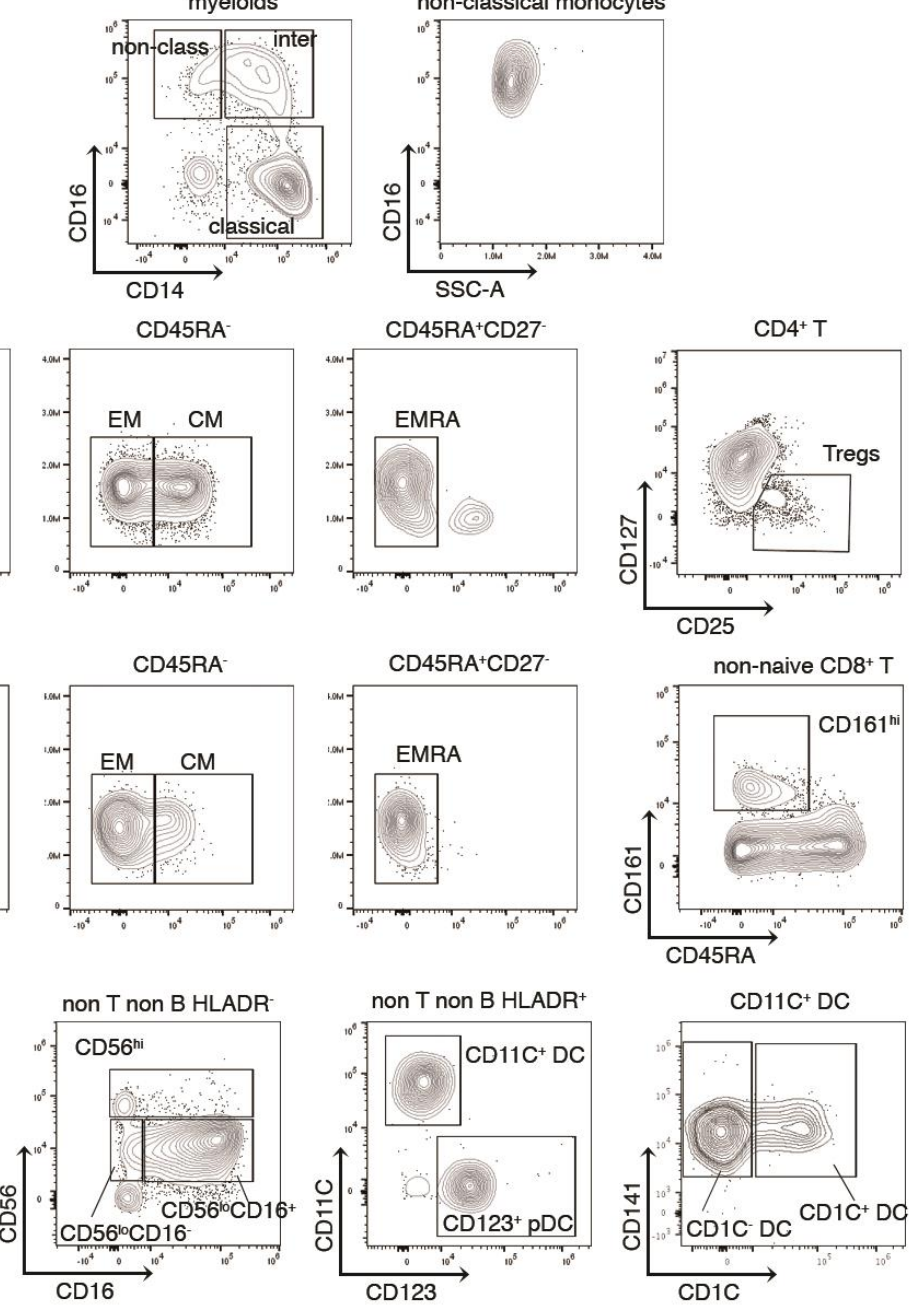

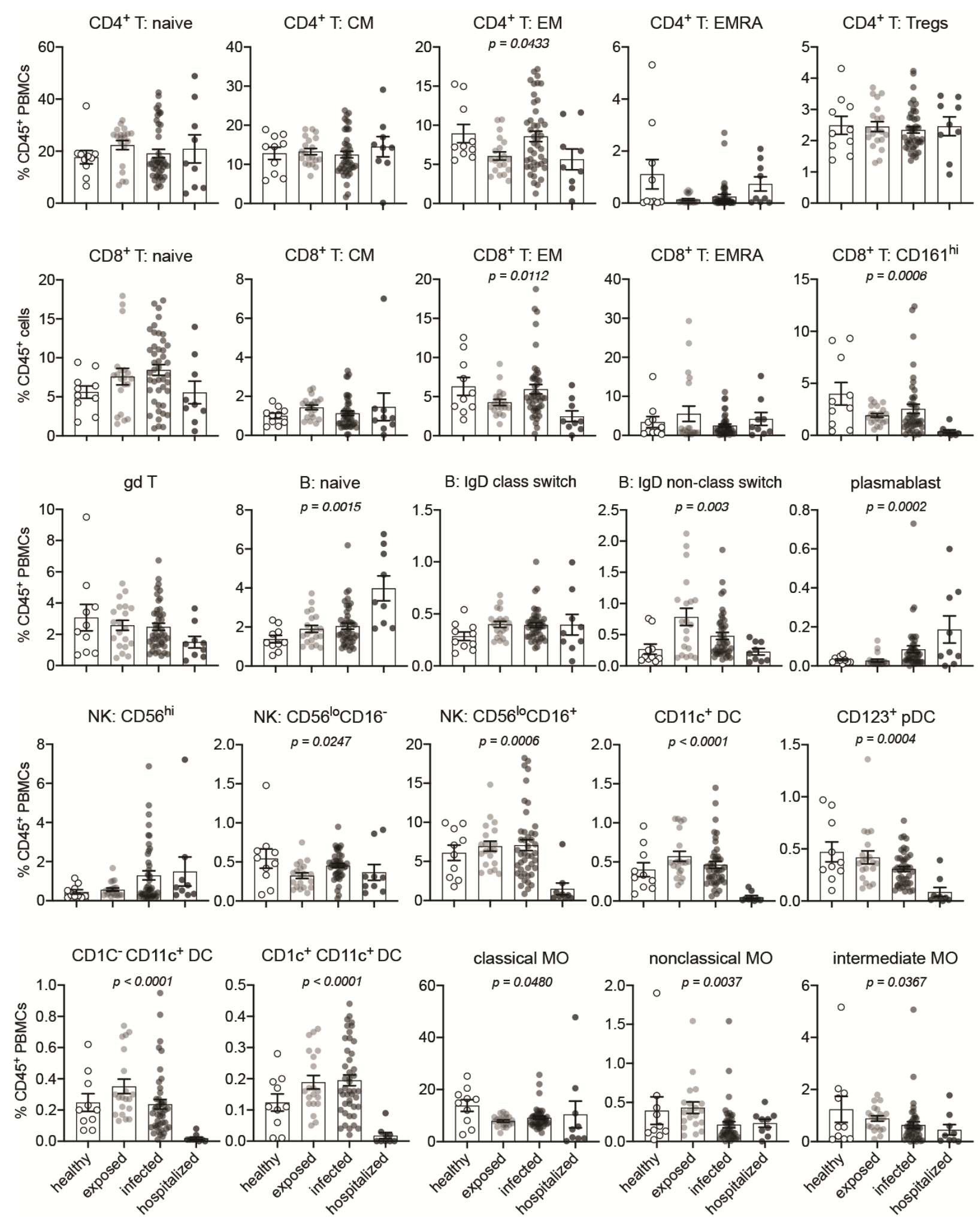
bioRxiv preprint dol: https://doi.org/10.1101/2020.12.01.407148; this version posted December 1, 2020. The copyright holder for this preprint (which was not certified by peer review) is the author/funder, who has granted bioRxiv a license to display the preprint in perpetuity. It is made available under aCC-BY-NC-ND 4.0 International license.

638 Supplementary Figure S3

A live singlets

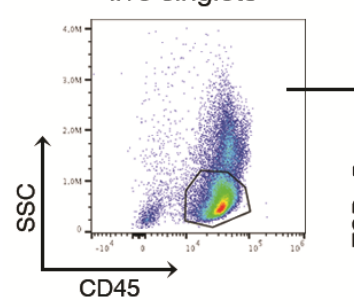

B
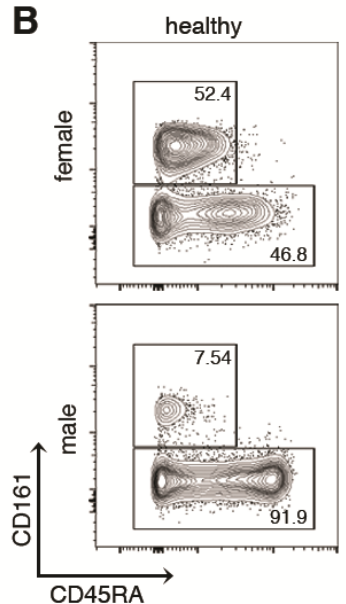

CD45+ lymphocytes
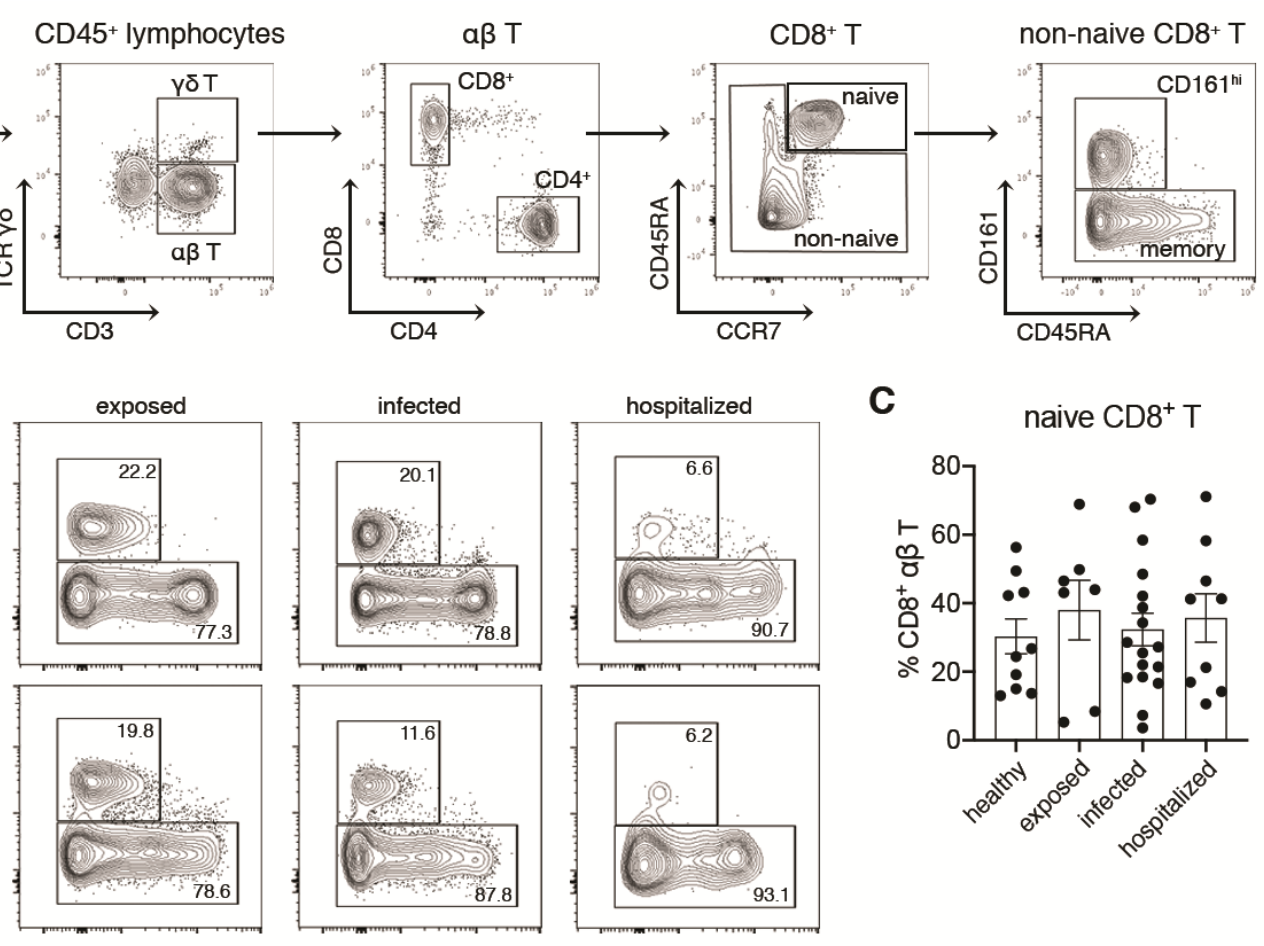

C naive $\mathrm{CD}^{+} \mathrm{T}$

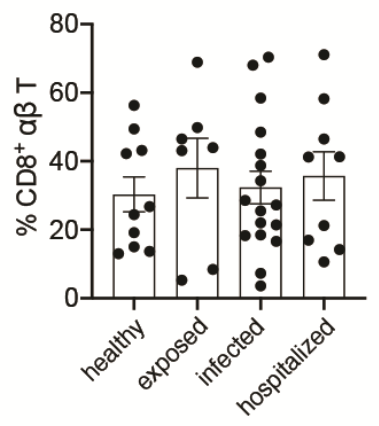

639 
bioRxiv preprint doi: https://doi.org/10.1101/2020.12.01.407148; this version posted December 1, 2020. The copyright holder for this preprint (which was not certified by peer review) is the author/funder, who has granted bioRxiv a license to display the preprint in perpetuity. It is made available under aCC-BY-NC-ND 4.0 International license.

\section{Supplementary Figure S4}

A

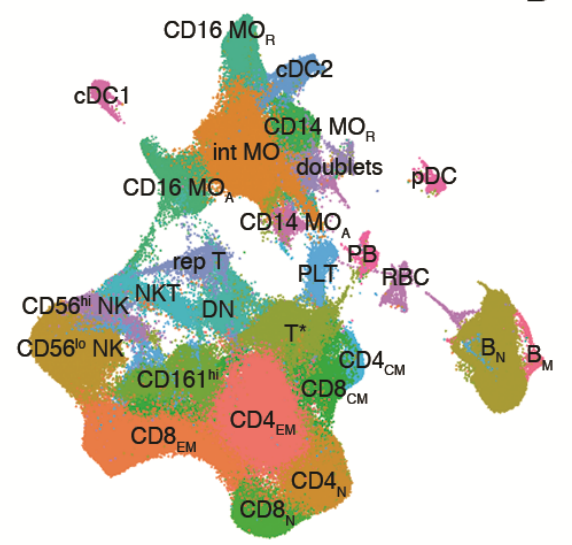

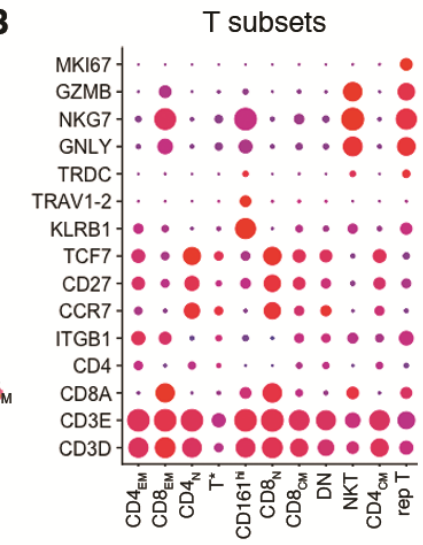

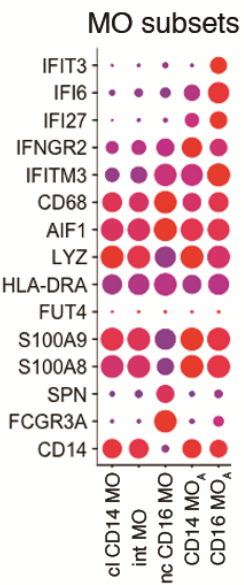

NK, B, DC and others

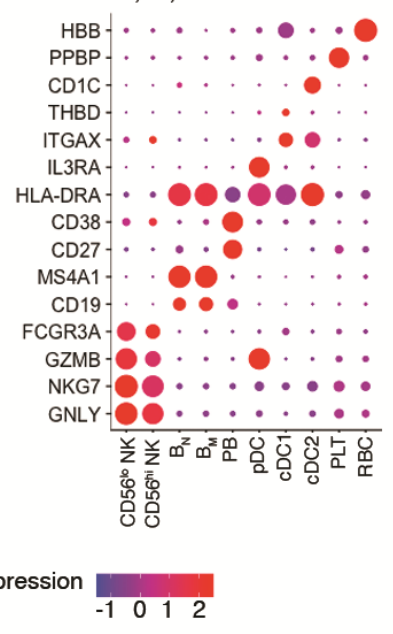


bioRxiv preprint doi: https://doi.org/10.1101/2020.12.01.407148; this version posted December 1, 2020. The copyright holder for this preprint (which was not certified by peer review) is the author/funder, who has granted bioRxiv a license to display the preprint in perpetuity. It is made available under aCC-BY-NC-ND 4.0 International license.

\section{Supplementary Figure S5}
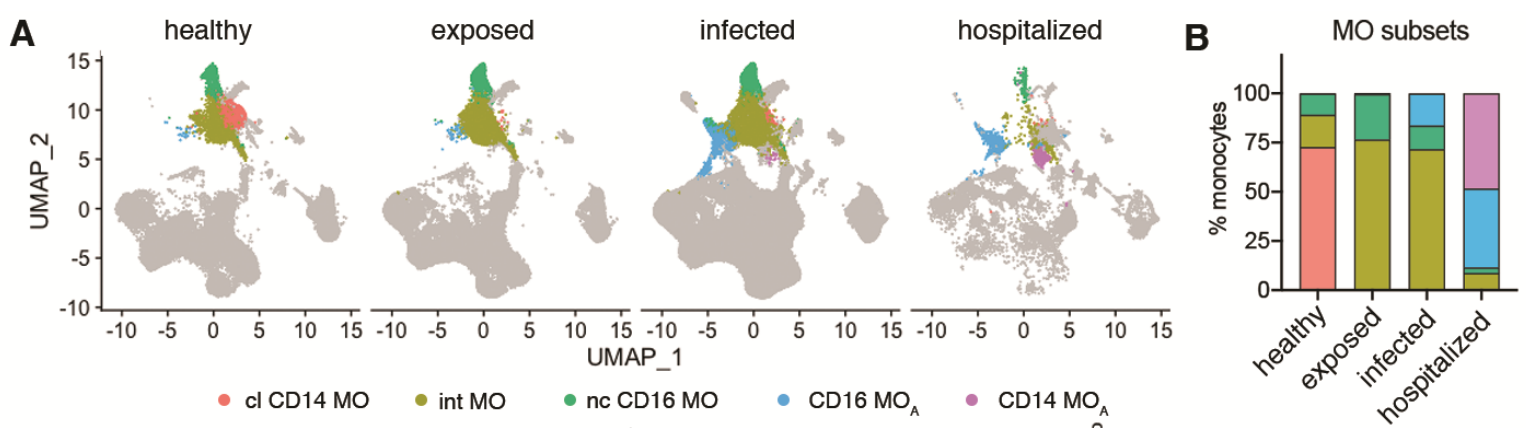

C
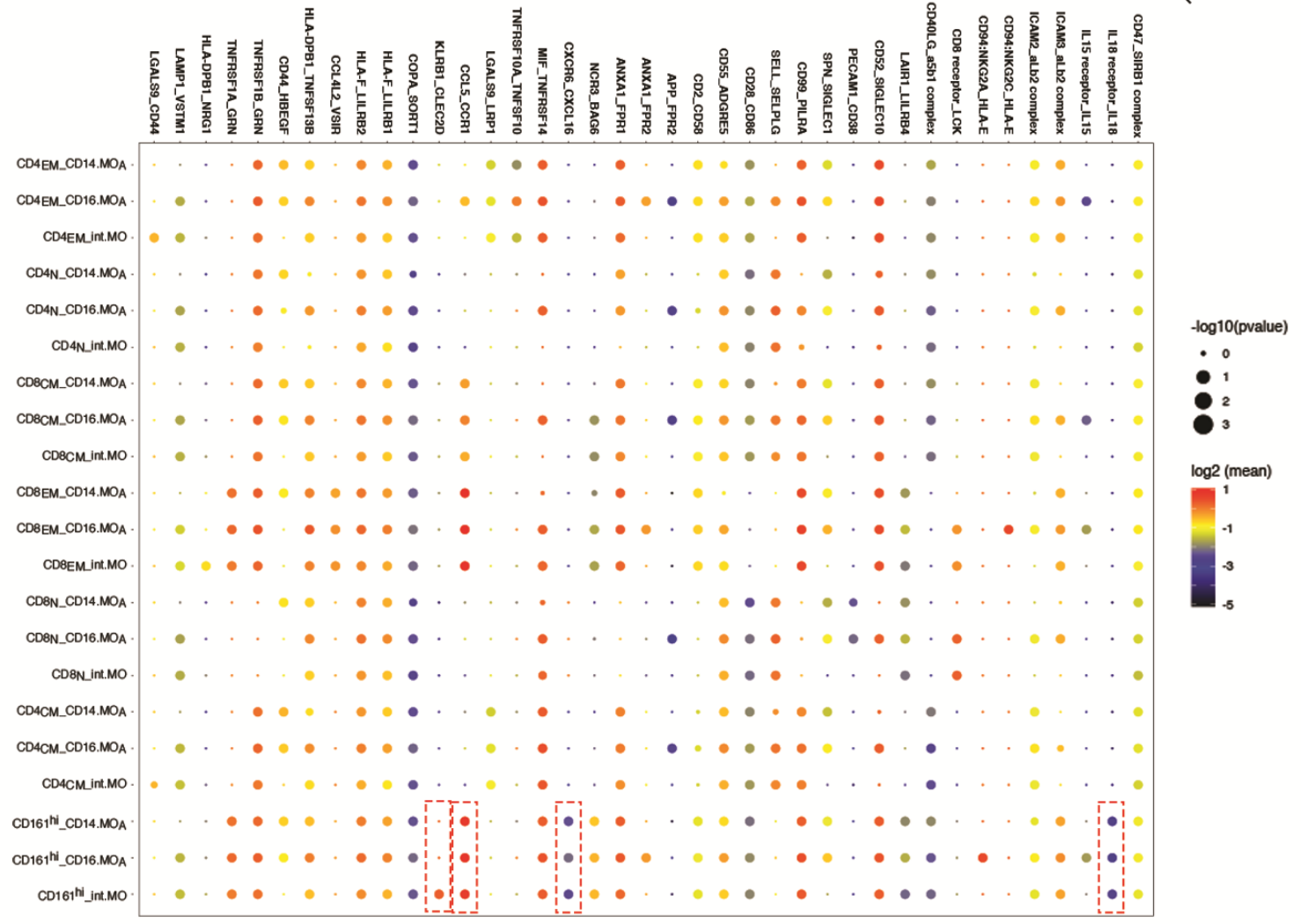

D

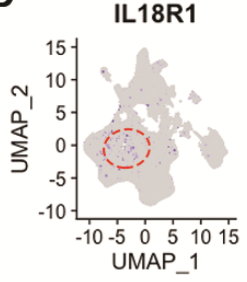

IL18

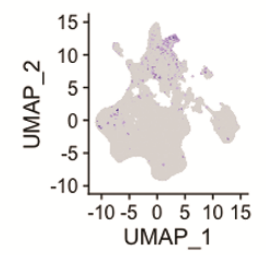

CXCR6

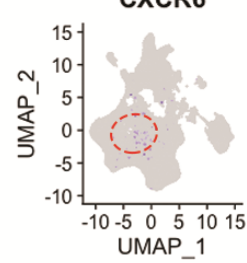

CXCL16

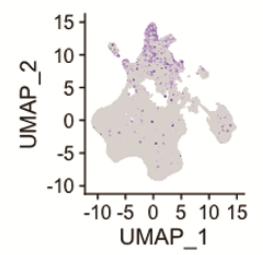

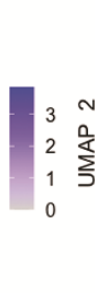

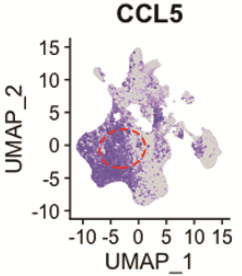

CCR1
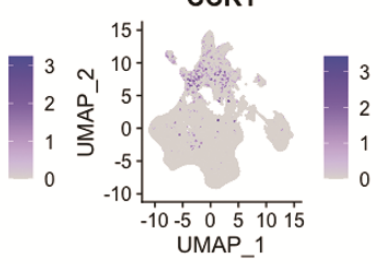

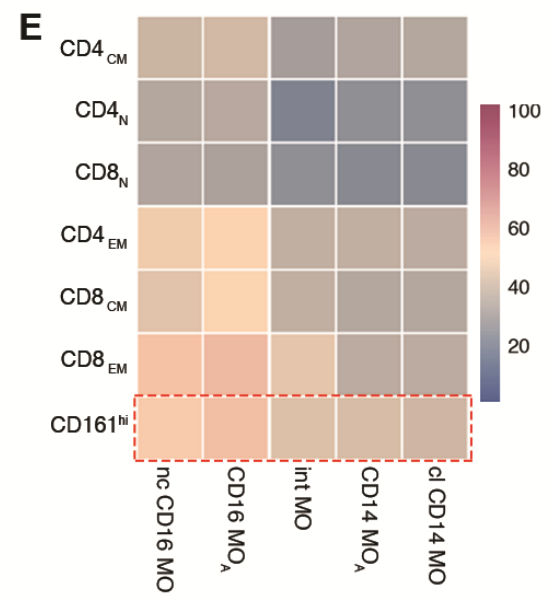


bioRxiv preprint doi: https://doi.org/10.1101/2020.12 01.407148; this version posted December 1,2020 . The copyright holder for this preprint (which was not certified by peer review) is the author/funder, who has granted bioRxiv a license to display the preprint in perpetuity. It is made available under aCC-BY-NC-ND 4.0 International license.

\section{Supplementary Figure S6}

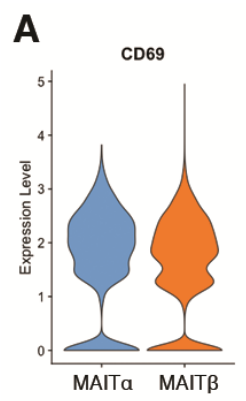

B

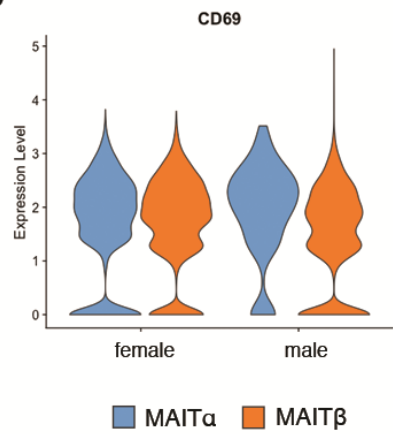

C

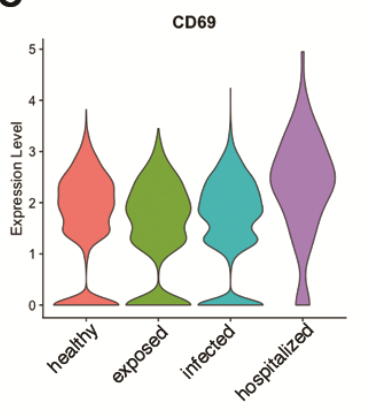

D

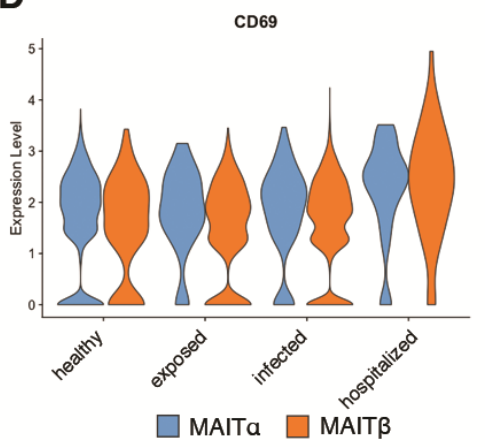




\section{Supplementary Figure S7}
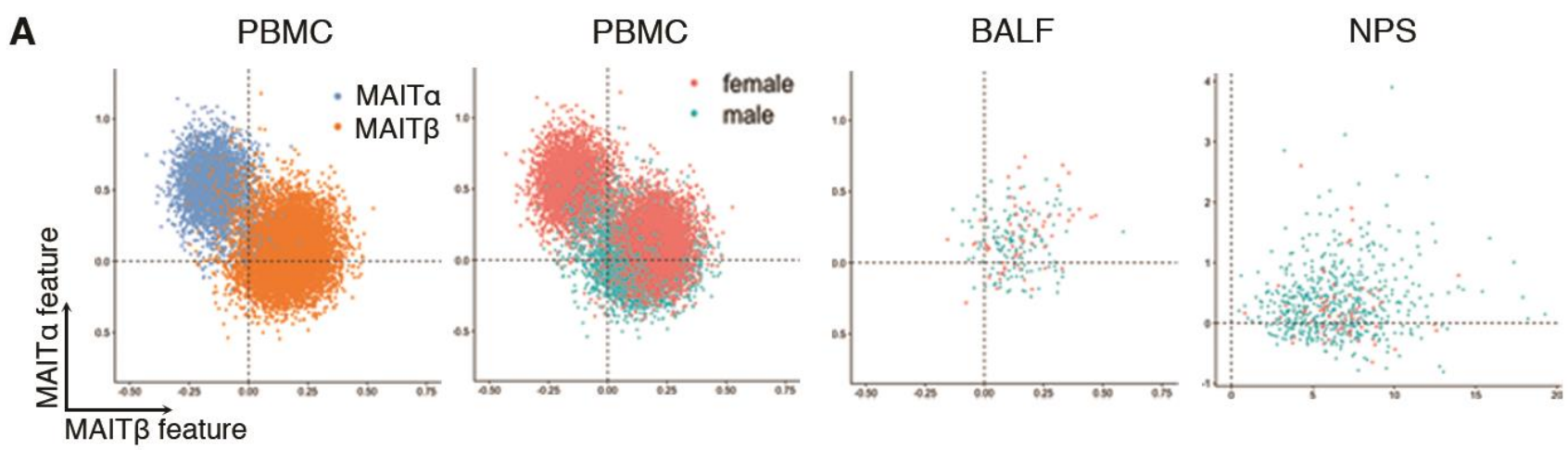

B

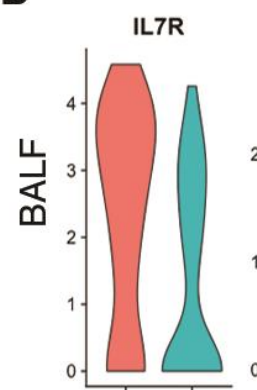

IL2RG

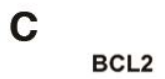

FOXP1
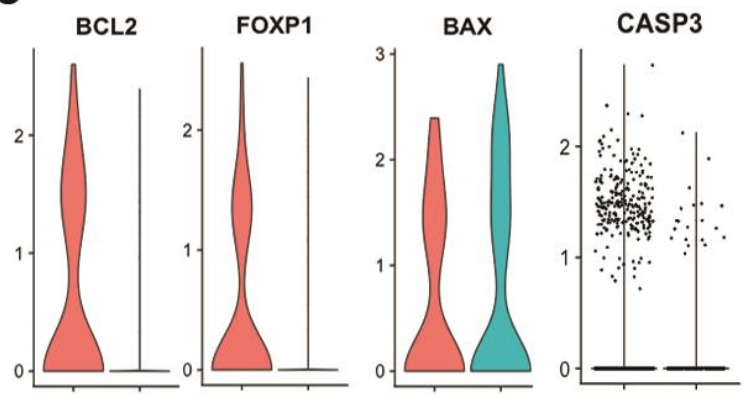

D
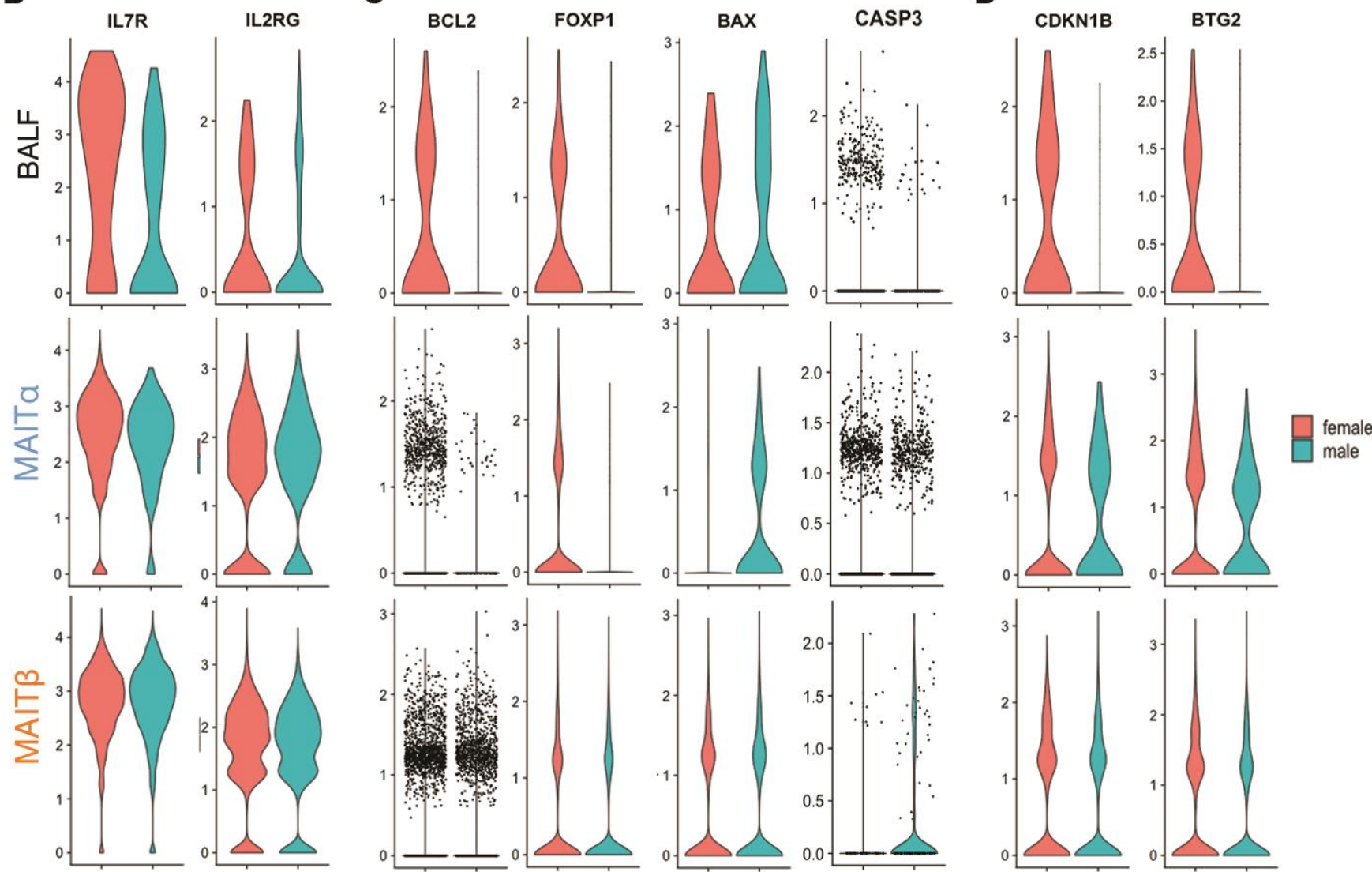